\title{
Atmospheric Circulation of Close-In Extrasolar Giant Planets: I. Global, Barotropic, Adiabatic Simulations
}

\author{
James Y-K. Cho, \\ Department of Terrestrial Magnetism, Carnegie Institution of Washington, \\ 5241 Broad Branch Road, N.W., Washington, D.C. 20015, USA \\ and \\ Astronomy Unit, School of Mathematical Sciences, Queen Mary, University of London, \\ Mile End Road, London E1 $4 N S$, UK \\ Kristen Menou, \\ Department of Astronomy, Columbia University, \\ 550 W. 120th Street, New York, NY 10027, USA \\ Bradley M. S. Hansen, \\ Department of Physics and Astronomy and Institute for Geophysics and Planetary Physics, \\ University of California, 475 Portola Plaza, Box 951547, Los Angeles, CA 90095, USA \\ and \\ Sara Seager \\ Department of Terrestrial Magnetism, Carnegie Institution of Washington, \\ 5241 Broad Branch Road, N.W., Washington, D.C. 20015, USA
}

\begin{abstract}
We present results from a set of over 300 pseudospectral simulations of atmospheric circulation on extrasolar giant planets with circular orbits. The simulations are of high enough resolution (up to 341 total and sectoral modes) to resolve small-scale eddies and waves, required for reasonable physical accuracy. In this work, we focus on the global circulation pattern that emerges in a shallow, "equivalent-barotropic", turbulent atmosphere on both tidally synchronized and unsynchronized planets. A full exploration of the large physical and numerical parameter-space is performed to identify robust features of the circulation. The model is validated with Solar System giant planets. For extrasolar giant planets with physical parameters similar to HD 209458 b - a presumably synchronized extrasolar giant planet representative in many dynamical respects - the circulation is characterized by the following features: 1) a coherent polar vortex that revolves around the pole in each hemisphere; 2) a low number - typically two or three of slowly-varying, broad zonal (east-west) jets that form when the maximum jet speed
\end{abstract}


is comparable to, or somewhat stronger than, those observed on the planets in the Solar System; and, 3) motion-associated temperature field, whose detectability and variability depend on the strength of the net heating rate and the global root mean square wind speed in the atmosphere. In many ways, the global circulation is Earth-like, rather than Jupiter-like. However, if extrasolar giant planets rotate faster and are not close-in (therefore not synchronized), their circulations become more Jupiter-like.

subject headings: planetary systems - planets and satellites: general - stars: atmospheres turbulence

\section{Introduction}

A large number of extrasolar planets orbiting nearby sun-like stars is now known. ${ }^{1}$ So far, all of the planets are giant planets, which orbit $\lesssim 5 \mathrm{AU}$ from their host stars. These extrasolar giant planets possess very interesting and unexpected orbital properties. Some of them orbit extremely close to their host stars (semi-major axis $a \lesssim 0.1 \mathrm{AU}$ ) and have very nearly circular orbits (eccentricity $e \lesssim 0.05$ ). These are the close-in extrasolar giant planets. If they possess tidal $Q$ parameter values similar to that of Jupiter $\left(\sim 10^{6}\right)$, they are expected to be in 1:1 spin-orbit resonance, or synchronous, states (Goldreich and Soter 1966; Rasio et al. 1996; Lubow, Tout, \& Livio 1997; Ogilvie \& Lin 2004). The rest of the extrasolar giant planets orbit further away from their host stars, generally with substantial eccentricities. Among them, some have nearly circular orbits however, like the Solar System giant planets. In this paper, we focus our study on the atmospheric circulation of all giant planets with circular orbits - from the close-in extrasolar giant planets to the more distant "unsynchronized" extrasolar giant planets, as well as the Solar System giant planets.

The existence of a dynamic, stably-stratified (radiative) outer layer is a common feature on planets with atmospheres. Extrasolar giant planets are also expected to possess such layersindependent of the proximity to their host stars. The dynamic layer is typically $\lesssim 10$ pressure scale heights thin, much smaller than the radius of the planet. It extends from just below the "weather layer" (at the bottom) up to the turbopause (at the top)-i.e., from near the top of the convection zone up to the tenuous region where molecular diffusion begins to dominate over advection. Following the first detections of secondary eclipses for three transiting extrasolar giant planets by the Spitzer Space Telescope (Charbonneau et al. 2005; Deming et al. 2005, 2006), the radiative layers of extrasolar giant planets are now the focus of intense observational and theoretical efforts (Seager et al. 2005; Barman, Hauschildt \& Allard 2005; Burrows, Hubeny \& Sudarsky 2005; Burrows, Sudarsky \& Hubeny 2006; Fortney et al. 2005; Iro et al. 2005; Williams et al. 2006). Since vigorous motions and meteorology in these layers will strongly influence observable properties, a good understanding of atmospheric dynamics on extrasolar giant planets is needed.

\footnotetext{
${ }^{1}$ See, e.g., http://exoplanets.org/almanacframe.html and http://www.obspm.fr/encycl/encycl.html.
} 
The understanding is especially crucial for the synchronized planets with their unique heating configuration. It is uncertain what temperature distribution should result from the combined effects of stationary dayside heating and advection on the atmospheres of these planets (e.g. Showman and Guillot 2002; Cho et al. 2003). This is partly because they are dynamically very different from the Solar System giant planets (Cho et al. 2003; Menou et al. 2003). Close-in extrasolar giant planets are also independently important for theory since their expected synchronous states provide an idealized forcing situation, important for improving understanding of planetary (as well as possibly stellar) atmospheres in general.

Recently, several global simulations of close-in extrasolar giant planet atmospheric circulation have been performed, focusing on different aspects of the overall complex problem (Showman and Guillot 2002; Cho et al. 2003; Cooper and Showman 2005). Global calculations are necessary to study the full effect of rotation on large-scale ${ }^{2}$ flows - the general circulation - on planets. Cho et al. (2003) have carried out a high-resolution, one-layer turbulence simulation of the close-in extrasolar giant planet HD $209458 \mathrm{~b}$ using the pseudospectral method. They stress that the gross feature of the atmospheric flow on this "Hot Jupiter" is markedly different from that on the Jupiter: the horizontal circulation pattern is actually more like that of the Earth or Venus, near and above the cloudtops. As elucidated in the companion paper by Menou et al. (2003), this is expected on dynamical grounds. The marked difference is due to the close-in planet's slower rotation rate $\left(\Omega^{\mathrm{HD}} \approx 2.1 \times 10^{-5} \mathrm{rad} \mathrm{s}^{-1}\right)$ and hotter equilibrium temperature $\left(T_{e}^{\mathrm{HD}} \sim 1500 \mathrm{~K}\right)$, compared to those of Jupiter $\left(\Omega^{\mathrm{Jup}}=1.4 \times 10^{-4} \mathrm{rad} \mathrm{s}^{-1}\right.$ and $\left.T_{e}^{\mathrm{Jup}}=130 \mathrm{~K}\right)$. Showman and Guillot (2002) and Cooper and Showman (2005) have also performed global simulations. Using the finite difference method in multiple-layer models, they focus on the laminar state and the vertical structure of the circulation. Both of these multiple-layer studies solve a more general set of equations than that of Cho et al. (2003), allowing vertical variations over many pressure scale heights to be represented. However, the multiple-layer studies are more idealized in their model setup: an atmosphere at rest is heated, leaving out the important influences of the jet streams and turbulent eddies that would in reality be present from the outset.

The structure and robustness of the flow forming under applied heating is the primary subject of this paper. Specifically, global circulation patterns possible in a stably-stratified, turbulent atmosphere on extrasolar giant planets with circular orbits are studied. This paper presents several much-needed developments in extrasolar planet characterization study: 1) a broad parameterspace exploration of a common type of extrasolar giant planet-i.e., extrasolar giant planets with circular orbits, which include the transiting close-in extrasolar giant planets; 2) a validation of the circulation model against a previously successful giant planet circulation model; and, 3) a detailed description of the employed model so that equitable comparisons can be made with alternative flow models. As in Cho et al. (2003), we here use the "equivalent-barotropic formulation" (Salby 1989) of the primitive equations of meteorology (cf., Holton (1992) and Salby (1996)), the equivalent-

\footnotetext{
${ }^{2}$ By "large-scale", it is meant horizontal scale $L \gtrsim R_{p} / 10$, where $R_{p}$ is the radius of the planet.
} 
barotropic equations. The equivalent-barotropic equations allow us to practically perform the large number of high-resolution simulations necessary for the exploration while bypassing the issue of the current lack of information on the vertical structure (e.g., distribution of radiatively-active species). The equivalent-barotropic equations, in the form used in this paper, also allow a physically clear interpretation of heating and cooling associated with dynamics in a stratified layer. In this paper, we focus on the adiabatic dynamics - appropriate for dynamical timescales shorter than the radiative timescales, or equivalently when the diabatic heating and cooling nearly cancel in the net. The focus sets the stage for diabatic (non-adiabatic) calculations (Cho et al., in preparation).

The overall plan of the paper is as follows. In $\S 2$, we briefly describe the current state of understanding of giant planet atmospheric circulation, laying stress on the aspects that are important for extrasolar giant planets. In $\S 3$, we present the primitive equations, the equations that govern the large-scale dynamics of atmospheres, and discuss the origins and properties of the reduced version of the primitive equations used, the equivalent-barotropic equations. Readers wishing more details on the derivation of the equivalent-barotropic equations are referred to Salby (1989). Readers familiar with the equations of atmospheric motion may skip the early part of this section. Later in the same section, we describe our representation of the differential thermal forcing, important for modeling close-in extrasolar giant planet atmospheres. In $\S 4$, we present the simulations of Solar System giant planets using the equivalent-barotropic equations. The simulations provide model validation as well as an initial assessment of circulation on unsynchronized extrasolar giant planets, which are essentially modeled in the present work as Solar System giant planets with greater equilibrium temperature $T_{e}$ and/or slower rotation rate $\Omega$. In $\S 5$, we summarize the detailed parameter-space exploration of the circulation on HD 209458 b, a representative close-in extrasolar giant planet in many dynamical aspects. This brackets circulations possible on close-in extrasolar giant planets in the context of one-layer modeling. In $\S 6$, we conclude the description of our global adiabatic calculations.

\section{Atmospheric Circulation on Giant Planets}

The dynamically-active layer of a giant planet atmosphere is forced by agents both external and internal to the layer, which render the large-scale flow in the layer very complex. The forcing comes from $i$ ) the host star irradiation above, $i$ ) the convection below, and iii) the momentum and heat fluxes within. The impinging stellar radiation is mostly shortwave (i.e. ultraviolet and visible). Some of the incoming radiation is scattered by the atmospheric gases, which is composed mostly of inert $\mathrm{H}_{2}$ and $\mathrm{He}$ by volume. Some of it is reflected back by clouds, if they are present. And, some of it is absorbed by the atmosphere, particularly by active minor constituents such as $\mathrm{H}_{2} \mathrm{O}$ vapor and clouds. The atmospheric gases and clouds also emit and absorb longwave (i.e. infrared) radiation, leading to either further heat transfer between different parts of the atmosphere or heat loss to space. Convection provides direct stirring as well as latent heat from condensation. Roughly speaking, the static atmospheric structure is in radiative equilibrium above the weather layer (in 
the middle and upper part of the active layer) and in radiative-convective equilibrium below the weather layer (near the bottom of the active layer). In this way, radiative transfer processes are important in establishing the vertical temperature structure throughout the active layer. However, dynamical processes (such as transport and dissipation of eddies ${ }^{3}$ and waves) significantly modify the basic temperature structure that would be established by the radiative processes alone, in the absence of motion.

On Solar System giant planets, despite the markedly different orbital, thermal, and chemical properties, the atmospheres all exhibit strong zonal (east-west) banding and associated zonal jets (longitudinally-averaged eastward winds), with long-lasting vortices embedded in them. The bands, jets, and vortices can all be understood as a dynamical equilibrium state of a stably-stratified shallow layer of turbulent fluid on a rotating sphere (Cho and Polvani 1996a). In a stratified-rotating fluid, the motion is predominantly layer-wise and two-dimensional (2-D). As a consequence, the flow exhibits a simultaneous inverse cascade of energy up to the large scales and forward cascade of enstrophy $^{4}$ down to the small scales (Kraichnan 1967; Leith 1968; Batchelor 1969). This is in marked contrast to the usual forward cascade of energy (only) in 3-D turbulence (Kolmogorov 1941a,b,c). In 2-D turbulence, the inverse cascade roughly corresponds to growth of eddies (vortices) by successive mergers (McWilliams 1984). In an isotropic condition, the eddies ultimately grow to the size of the domain. On the sphere, however, a fundamental anisotropy due to the geometry is present, if the sphere is rotating: Coriolis acceleration provides a restoring force on the growing eddies in the meridional (north-south) direction while none in the zonal direction. In this anisotropic condition, eddies are free to grow to the largest available scale in the zonal direction but restricted in the meridional direction - thus forming the bands and jets (Rhines 1975). In this heuristic explanation of the bands, the characteristic meridional width of the bands is loosely defined by the Rhines scale, $L_{\beta} \equiv \pi(2 U / \beta)^{1 / 2}$, where $U$ is the root mean square wind speed of the jets and $\beta \equiv 2 \Omega \cos \phi / R_{p}$ with $\phi$ the latitude and $R_{p}$ the radius of the planet. According to this mechanism, the number of jets and bands is $\approx \pi R_{p} / L_{\beta}$ and is an increasing function of the planetary rotation rate $\Omega$.

Cho and Polvani (1996b) have explicitly shown in numerical simulations that the banded structures on all Solar System giant planets can be reproduced very well through this " $\beta$-mechanism". In their study, they use the shallow-water equations, which formally applies to a thin layer of water with a free surface (Pedlosky 1987). The shallow-water simulations capture reasonably well the number, size, and amplitude of the zonal jets - the gross features of the atmosphere - on all four giant planets of the Solar System. In addition, the model captures the anticyclonic ${ }^{5}$ dominance on

\footnotetext{
3 "Eddies" are the residual of the dynamical field after the subtraction of the mean. In geophysical fluid dynamics, "eddies" are often used interchangeably with vortices and even waves.

${ }^{4}$ Enstrophy is the "vortical energy" of the fluid, defined by $\frac{1}{2} \zeta^{2}$, where $\vec{\zeta}=\zeta \mathbf{k}=\nabla \times \mathbf{v}$ is the vorticity (parallel to the vertical direction $\mathbf{k}$ ) and $\mathbf{v} \in \Re^{2}$ is the horizontal velocity.

${ }^{5}$ Cyclones (anticyclones) are vortices defined by the positive (negative) sign of $\vec{\zeta} \cdot \boldsymbol{\Omega}$, where $\boldsymbol{\Omega}$ is the planetary rotation vector (which defines the north pole).
} 
Jupiter (Mac Low and Ingersoll 1986). It is important to note that their simulations use only the observed physical parameters values of $\Omega, g, R_{p}, \bar{U}$, and $T_{e}$ (planetary rotation rate, surface gravity, planetary radius, global root mean square wind speed, and equilibrium temperature, respectively). Thus, the predictions of the simulations are essentially "cold predictions" since no presumed information on the vertical structure or the type of forcing has been used. The qualitative, good match obtained between the simulations and the observations gives confidence in the basic approach of using a barotropic, adiabatic, shallow-layer turbulence model to study giant planet atmospheric circulations. An alternative model of bands and jets on a giant planet also exists. This model is based on the surface expression of putative deep, convective, Taylor-Proudman columns aligned with the planetary rotation vector $\boldsymbol{\Omega}$ (cf., Sun et al. (1993), and references therein). However, to date, the shallow-water model is the only model that is able to reproduce the global circulation patterns of all four Solar System giant planets. Recently, Heimpel et al. (2005) have also obtained a good match to Jupiter's surface zonal wind structure using a 3-D global convection model. Their calculation is performed in a thin spherical zone, which in principle is similar to the shallow layer turbulence approach (rather than the deep convection laminar approach).

Given the success of the shallow-water model ${ }^{6}$, it is natural to try and extend the approach to extrasolar giant planets. However, a number of issues critical for the study of extrasolar giant planet atmospheric dynamics is currently either poorly understood or await observational constraints. This entails that current extrasolar planet atmosphere models, including radiative transfer models, necessarily contain some basic and crucial unknowns. The vertical distribution of active species has already been mentioned; $\Omega$ is another. The latter is crucial in characterizing the planet's visual, spectral, and hydrodynamic properties (Cho and Polvani 1996b; Showman and Guillot 2002; Cho et al. 2003; Menou et al. 2003). Since orbital periods can be accurately measured, the rotation rate of close-in extrasolar giant planets can at least be inferred by assuming synchronization. However, even in this case, there is still the question of how to initialize the model calculation. A background zonal flow, for example, will nontrivially affect the evolution of any developing flow by nonlinearly interacting with it. Without adequate initial and boundary conditions, it cannot be stressed enough that current model calculations should more properly be considered as revealing important mechanisms and plausible flow states, rather than predicting reality.

With the above caveats in mind, we forge ahead by identifying giant planet properties that should be general. Like the Solar System giant planets, extrasolar giant planets may have been initially rotating rapidly at a large distance from their parent stars. Many of them may have subsequently migrated to smaller orbital radii, resulting in the observed population of extrasolar giant planets. From the point of view of atmospheric circulation (which is established on timescales very much shorter than the evolutionary and migration timescales), the implication is that the circulation regime would be a slow function of time under increasing levels of stellar irradiation (absent

\footnotetext{
${ }^{6}$ We must keep in mind, however, the gross simplifications inherent in the shallow-water model (see $\S 3$ ). The model does not - and in many cases cannot - address a number of important aspects about the circulation.
} 
complications due to catastrophic events, such as giant impacts). Hence, if an extrasolar giant planet's circulation regime were Jupiter-like initially, it need not be so over time. Of particular interest here is that planets reaching small enough orbital distances for tidal circularization and synchronization to operate will have a pre-established circulation pattern "on arrival" to the synchronization region. Although the detailed sequence of events is difficult to characterize, it is worth emphasizing that it may be crucially important to account for this in atmospheric simulations: the planets make a transition from a zonally-symmetric (diurnally-averaged) to a zonally-asymmetric (dayside-only) heated state while a pre-existing circulation pattern is already established in their atmospheres.

Throughout this evolutionary sequence, the stably-stratified layers on the planets experience heating from both the central star and the convective interior. An accurate model of the layers must include minimally the heating effects from both sources - more precisely, the net heating from the two. For the vertical direction, this is addressed by a radiative-convective equilibrium model, which takes into account convective instability by adjusting the temperature profile to a neutrally stable profile in the unstable region. However, because it is still a static model, accounting for only the vertical and non-mechanical transfer of energy, it predicts an equator-pole temperature gradient that is in general much larger than the actual, observed state. This is found to be the case for all Solar System planets with an atmosphere. If the actual gradient is such that the planet experiences net heating (i.e., the actual temperature is lower than the equilibrium temperature) at low latitudes while negative net heating (i.e., cooling) at high latitudes (actual temperature higher than equilibrium temperature) - as is observed on the Earth, for example - thermal equilibrium requires that the imbalance be compensated by a net poleward transfer of heat. When balanced by atmospheric motions, the situation is in a "radiative-dynamical" equilibrium.

It is important to understand that the flow in the radiative layer is generally not driven by the stellar heating directly but by the deviation from the equilibrium temperature. Moreover, the deviation responsible for the transport is caused by the atmospheric dynamics - specifically, by eddies and waves. Hence, it is more proper to think of the dynamics as controlling the temperature distribution, rather than the other way around. This is why it is crucial to represent the flow dynamics properly, both initially and throughout the flow evolution. Well-known examples of eddy/wave-induced temperature and flow modifications exist. Rossby ("planetary") and gravity (buoyancy) waves cause major warmings over the cold pole, forcing the region to be above the radiative equilibrium temperature and undergo cooling. Waves can also induce quasi-periodic flow, and its associated temperature reversals, in the equatorial region. Cold regions at the subsolar point just above the troposphere and at the top of the radiative layer are eddy/wave-driven as well, as the rising air in those regions cool adiabatically. These are fairly well understood phenomena in the Earth's atmosphere, where considerable observational data is available (cf., Andrews et al. (1987)). Remarkably, similar heating and cooling mechanisms appear likely to operate on extrasolar giant planets, as we shall show later ( $\$ 4$ and $\S 5)$. On rapidly-rotating and unsynchronized extrasolar giant planets, meridional transfer of heat is expected; and, on slowly-rotating or synchronized extrasolar 
giant planets, zonal transfer of heat is expected, in addition to the meridional transfer.

Under certain circumstances, the Newtonian cooling approximation may be fruitfully used to represent the net heating (cf., Andrews et al. (1987) and Salby (1996)). The approximation is a simple representation of the radiative effects on the large-scale dynamics - a relaxation of the temperature field to a specified "equilibrium" distribution. The equilibrium distribution, which depends in a complicated way on the atmospheric constituents and their spatial distribution, is not known a priori in general. The approximation is valid when the timescale of motion, $\tau_{a} \sim L / U$, is shorter than the timescale for radiative cooling, $\tau_{r} \sim \rho c_{p}\left(d \mathcal{T} / d z^{*}\right)^{-1} /\left(4 \sigma T^{3}\right)$. Here, $L, U, p$, $c_{p}, \sigma, T, \mathcal{T}$, and $z^{*}$ are the characteristic length, characteristic speed, basic density, specific heat at constant pressure, Stefan-Boltzmann constant, equilibrium temperature, transmission function, and $\log$-pressure height $\left(=H_{p} \ln \left(p_{\text {ref }} / p\right)\right.$, with $H_{p}$ the pressure scale height and $P_{\text {ref }}$ a reference pressure), respectively.

Note that the Newtonian approximation is a crude estimate: it ignores vertical motion $(d p / d t)$, assumes maximum temperature perturbation $T^{\prime}$ small compared to the equilibrium temperature (i.e., $T^{\prime} \ll 1500 \mathrm{~K}$ for close-in extrasolar giant planets), approximates the vertical gradient of the transmission function to be $\sim 1 / H_{p}$, and requires the environment to be in local thermodynamic equilibrium condition (cf., Andrews et al. (1987)). Caution should be exercised when extending its use to extrasolar giant planets. The approximation should be valid in the deeper $(\sim 1$ to $\sim 10$ bar $)$ levels or cooler $\left(\lesssim 10^{3} \mathrm{~K}\right.$ ) regions of atmosphere, where $\tau_{a} \ll \tau_{r}$. At higher levels, the $\tau_{a} \ll \tau_{r}$ condition is not met, since $\rho$ and $p$ decreases with height. Moreover, if heat is efficiently mixed, the condition may not be uniformly met on the night side of synchronized planets as well. Even on the much cooler Earth $(T \sim 255 \mathrm{~K})$, the approximation is not globally valid. It works excellently in the upper troposphere ( $\sim 300$ mbar $)$ and reasonably well in the lower mesosphere $(\sim 100 \mathrm{mbar})$, but it fails strongly in the mid to upper mesosphere $(\$ 2 \mathrm{mbar})$ and at the tropopause $(\sim 200 \mathrm{mbar})$ and the mesopause ( $\sim 1$ mbar) (Andrews et al. 1987). Hence, the recent assertion by Iro et al. (2005), that they are able to distinguish - and by inference predict - $\tau_{r}$ to an accuracy better than a factor of 2 or 5 , is likely to be premature. This is because even if the "cool-to-space" term in the exchange integral in the radiative transfer calculation (cf., Andrews et al. (1987)) is somehow dominant all throughout the stratified layer and even if the gradient of the band transmission function were accurately known (which it is not), $\tau_{r}$ is very sensitive to $T\left(z^{*}\right)$, which is still poorly known.

In this paper, we focus on the dynamical implications of a specified temperature distribution with a steady day-night component on the circulation. In the context of the Newtonian cooling approximation, the situation corresponds to the adiabatic regime, where the conditions are not too far from radiative or radiative-convective equilibrium (without or with convective adjustment, respectively) or, equivalently, $\tau_{r} \gg \tau_{a}$. In actuality, based on preliminary calculations we have performed with Newtonian cooling, the cases when $\tau_{r}>\tau_{a}$ in our simulations are not qualitatively very different from the $\tau_{r} \gg \tau_{a}$ case - unless $\tau_{r} \sim \tau_{a}$. Hence, over a wide range, the $\tau_{r}$ dependence is not very strong. In the $\tau_{r} \ll \tau_{a}$ case, the dynamical equations cease to be physically instructive (though mathematically still interesting) when explicit Newtonian cooling is included, since $\tau_{a}$ is the 
timescale appropriate for structures described by the primitive equations. As an example, consider the 2 mbar level on HD 209458 b: if $\tau_{r} \sim 1$ hour, as is suggested by Iro et al. (2005) and assumed in Cooper and Showman (2005), flows obtained with explicitly-forced primitive equations should not be taken too seriously because the timescale for dynamics must be $\gg H_{p} / c_{s}$, where $c_{s}$ is the sound speed. Since $H_{p} / c_{s} \sim 0.1$ hour for HD $209458 \mathrm{~b}$, the smallest appropriate $\tau_{a}$ in explicitly-forced simulations is $\sim 1$ hour. For the $\tau_{r} \ll \tau_{a}$ case, the quasi-instantaneous radiative forcing may be more self-consistently modeled by imposing a fixed temperature structure in the simulations, since the forcing is fundamentally not being balanced by the slow, large-scale atmospheric motion. Numerically, the calculation blows up (unless extreme, unphysical dissipation is applied). Nevertheless, we have also studied the $\tau_{r} \lesssim \tau_{a}$ regime for completeness. A detailed description of the effects of $\tau_{r}$ on the dynamics and heat distribution will be described elsewhere.

\section{Circulation Model}

\subsection{The Primitive Equations}

The motion of an atmospheric fluid element is governed by the primitive equations. The equations are so called because they constitute the starting point for studying large-scale dynamics in the atmosphere. The presumption in their use is that the small scales, which are not resolvable either observationally or numerically, are parameterizable within the framework of large-scale dy-

namics. In atmospheric studies, $p$ is commonly used as the vertical coordinate, rather than the geometric height, $z=z(\mathbf{x}, p, t)$ with $\mathbf{x} \in \Re^{2} ; z$ becomes a dependent variable in this case. In the standard $p$-coordinates, the primitive equations are:

$$
\begin{aligned}
\frac{D \mathbf{v}}{D t}+\frac{u \tan \phi}{R_{p}} \mathbf{k} \times \mathbf{v} & =-\nabla_{p} \Phi-f \mathbf{k} \times \mathbf{v}+\mathcal{F}-\mathcal{D}, \\
\frac{\partial \Phi}{\partial p} & =-\alpha \\
\frac{\partial \omega}{\partial p} & =-\nabla_{p} \cdot \mathbf{v}, \\
\frac{D \theta}{D t} & =\frac{\theta}{c_{p} T} \dot{q}_{\text {net }},
\end{aligned}
$$

where

$$
\frac{D}{D t}=\frac{\partial}{\partial t}+\mathbf{v} \cdot \nabla_{p}+\omega \frac{\partial}{\partial p} .
$$

In Eq. (1), $\mathbf{v}(\mathbf{x}, t)=(u, v)$ is the (eastward, northward) velocity in a frame rotating with $\boldsymbol{\Omega}$, and $\Phi=g z$ is the geopotential, where $g$ is the gravitational acceleration (assumed constant and includes the contribution from the centrifugal acceleration) and $z$ is the height above a fiducial surface (e.g., the 1 bar pressure level); $\mathbf{k}$ is the unit vector normal to the planetary surface; $f=2 \Omega \sin \phi$ is the Coriolis parameter, the projection of the planetary vorticity vector $2 \boldsymbol{\Omega}$ onto $\mathbf{k} ; \nabla_{p}$ is the horizontal gradient on a constant- $p$ surface; $\omega \equiv D p / D t$ is the vertical velocity; $\alpha$ is the specific volume, the 
reciprocal of density $\rho ; \mathcal{F}$ and $\mathcal{D}$ represent the sources and sinks, respectively; $\theta=T\left(p_{\text {ref }} / p\right)^{\kappa}$ is the potential temperature ${ }^{7}$, where $p_{\text {ref }}$ is the reference pressure and $\kappa=R / c_{p}$ with $R$ the specific gas constant; and, $\dot{q}_{\text {net }}$ is the net diabatic heating rate. ${ }^{8}$ The above equations are closed with the ideal gas law, $p=\rho R T$, as the equation of state.

To arrive at Eq. (1), one begins with the full Navier-Stokes equations - along with the energy equation, equation of state, and boundary conditions - in the spherical geometry (Batchelor 1977). From the Navier-Stokes equations, two approximations are made at the outset. These are the "shallow atmosphere" and the "traditional" approximations (cf., Salby (1996)). The first assumes $z / R_{p} \ll 1$ and is valid for the stable layers on all giant planets, including the extrasolar giant planets. The second is formally valid in the limit of strong stratification, when the Prandtl ratio $(N / \Omega)^{2} \gg 1$. In the ratio,

$$
N(z)=\sqrt{\frac{g}{T}\left(\Gamma_{\mathrm{ad}}-\Gamma\right)}
$$

is the Brunt-Väisälä (buoyancy) frequency. In Eq. (2), $\Gamma_{\mathrm{ad}} \equiv g / c_{p}$ and $\Gamma \equiv-d T(z) / d z$ are the adiabatic and the environmental lapse rates, respectively (Pedlosky 1987). These approximations allow the horizontal (perpendicular to $\mathbf{k}$ ) Coriolis terms to be dropped from the Navier-Stokes equations and vertical accelerations to be assumed small, as will be further described below. As an example, consider HD 209458 b: if we assume Jupiter-like composition, temperature range of 100 to $\sim 1500 \mathrm{~K}$ across the globe, and nearly isothermal condition above the tropopause -i.e., where the temperature ceases to decrease significantly with altitude (cf., Seager and Sasselov (1998)), we obtain $(N / \Omega)^{2} \gtrsim 10^{4}$, where $N=g /\left(c_{p} T\right)^{1 / 2}$. The traditional approximation condition is also well met. Hence, as in many traditional atmospheric circulation studies, we may also take Eq. (1) as our starting point for extrasolar giant planets.

As can be seen in Eq. (1b), hydrostatic balance is assumed in the primitive equations. This condition restricts the vertical motion to be slow compared to the horizontal motion-or, equivalently, the vertical length scale of motions to be small compared to the horizontal length scale. It also allows local temperature to be associated with the atmospheric mass. That is, given two pressure surfaces $\left(p_{1}>p_{2}\right)$ bounding a vertical region, the local thickness (or mass per area) of the region, $\Delta z \equiv z_{2}-z_{1}$, is related to its average temperature via the hypsometric equation (Andrews et al. 1987):

$$
\Delta z=-H_{p}(\langle T\rangle) \ln \left(\frac{p_{2}}{p_{1}}\right)
$$

where $H_{p}=R\langle T\rangle / g$ is the local pressure scale-height and

\footnotetext{
${ }^{7}$ The potential temperature $\theta$ is related to the entropy $s$ by $d s=c_{p} d \ln \theta$.

${ }^{8}$ Note that various heating $(>0)$ and cooling $(<0)$ terms contribute to the diabatic heating rate $\dot{q}-$ e.g., stellar heating, longwave heating and cooling, latent heat, and sensible (conductive) heat. The Newtonian cooling scheme is one simple parameterization of $\dot{q}_{\text {net }}$.
} 


$$
\langle T\rangle=\left[\int_{p_{2}}^{p_{1}} d \ln p\right]^{-1} \int_{p_{2}}^{p_{1}} T(\mathbf{x}, t) d \ln p
$$

is the region's average temperature. Note that $\Delta z, H_{p}$, and $\langle T\rangle$ are all function of horizontal position and time. Eq. (3) is the basis of the pressure coordinate. Later, we will use an analog of Eq. (3) to obtain horizontal temperature distributions on extrasolar giant planets. This is accomplished through a more practical version of Eq. (1), which we now describe.

\subsection{Equivalent-Barotropic Formulation}

For many applications, Eq. (1) is much too unwieldy and broad in scope. Moreover, in the absence of observational information on the atmospheric vertical structure to constrain the many parameters needed to initialize and solve Eq. (1), a reduction of the equations is warrantedand necessary (cf., Andrews et al. (1987) and Pedlosky (1987)). A key element that must be represented faithfully when modeling the global circulation and thermal structure of planetary atmospheres is the dynamics of eddies and waves. Eddies and waves directly modify the flow and temperature structures on the large-scale by transporting momentum, heat, and radiatively-active species. However, the eddies and waves span from large scales down to scales well below those resolved by the current models solving the full primitive equations (Showman and Guillot 2002; Cooper and Showman 2005). A complete series of high-resolution 3-D calculations with all the key physics included and parameters explored is currently computationally prohibitive. Fortunately, some of this difficulty can be alleviated. In this work, we accomplish all of the reduction of the equations, resolving of the eddies and waves, and exploration of the parameter-space by vertically integrating Eq. (1) and using the well-tested and highly-accurate pseudospectral method (Orszag 1970; Eliassen et al. 1970) to solve the resulting, simpler set of equations. In doing so we are able to practically focus on some of the important elements critical for accurately capturing the global dynamics.

There are other justifications for focusing on the column-integrated, horizontal dynamics as well. First, the region of extrasolar giant planet atmosphere that requires modeling for current observations is an extremely thin layer within the overall stratified layer, at most a few scale heights thick and located near the tropopause. It is in this sub-layer where most of the stellar irradiation is seen to be absorbed in radiative transfer calculations (e.g. Seager et al. 2005; Iro et al. 2005) and from where the strongest (longwave) sensible features are likely to emerge observationally. In addition, given the nearly isothermal vertical structure in that region (e.g. Seager and Sasselov 1998; Sudarsky, Burrows \& Pinto 2000), the flow should be nearly vertically aligned. ${ }^{9}$ In atmospheric dynamics studies, the use of reduced vertical-resolution models is common - especially

\footnotetext{
${ }^{9}$ Actually, strong alignment should exist in the entirety of the stratified layer since the temperature structure is nearly isothermal throughout the layer.
} 
for the tropopause region and above, where there is a strong barotropic component (i.e. vertical alignment; cf., Juckes and McIntyre (1987); Salby et al. (1990); Polvani et al. (1995); Ferreira and Schubert (1997)). The typical length scale of the most unstable baroclinic mode (related to the non-alignment) is the Rossby deformation radius, $L_{\mathcal{R}}=N H / f \approx(g H)^{1 / 2} / f$, where $H \sim H_{p}$ is the characteristic thickness (Pedlosky 1987). For baroclinic waves to exert a strong presence, $L_{\mathcal{R}}$ should neither be $\ll R_{p}$ nor $\gg R_{p}$. As noted, the extreme smallness of $L_{\mathcal{R}}$ has already been exploited to great advantage in modeling Solar System giant planet circulations with a barotropic model (Cho and Polvani 1996b). For most unsynchronized extrasolar giant planets, we expect similar, or only slightly larger, values of $L_{\mathcal{R}}\left(\ll R_{p}\right)$. Even for close-in extrasolar giant planets, which receive intense heating, there is some justification for using a barotropic model since the $L_{\mathcal{R}}$ for them is generally $\gtrsim R_{p}$ (Cho et al. 2003; Menou et al. 2003).

\subsubsection{Equations}

Remarkably, Salby (1989) has shown that both adiabatic $\left(\dot{q}_{\text {net }}=0\right)$ and diabatic $\left(\dot{q}_{\text {net }} \neq 0\right)$ forms of Eq. (1) directly reduce to a set of equations similar to the shallow-water equations, a much studied set of equations in geophysical fluid dynamics. The shallow-water equations are the nonlinear version of the Laplace tidal equations (Laplace 1778; Gill 1982). Because these dynamical equations reveal the clearest interpretation of the role of heating in isentropic coordinates, we have used that coordinate form of the equations in this work. In isentropic coordinates, constant $\theta$-surfaces form the vertical coordinates, rather than $p$ - or $z$-surfaces. The new, reduced set of equations - the equivalent-barotropic equations in $\theta$-coordinates - are obtained by assuming that the constant surfaces of thermodynamic variables $(p, \rho, T, \theta)$ share a common horizontal structure. The salient feature of the equivalent-barotropic model, as well as the shallow-water model, is that they are currently the only global atmospheric dynamics models that can include the combined effects of differential rotation, stratification, and small-scale eddies and waves over long duration integrations. They are also currently the only model that can qualitatively reproduce the global circulation on giant planets "cold", without adjustable parameters and ad-hoc assumptions. In this paper, we present results for the adiabatic case only, in which the effect of heating is parameterized by deflecting the modeled surface consistently with the temperature at the surface (see below and §3.3). A forthcoming companion paper to this one will discuss the diabatic case (Cho et al., in preparation).

The equivalent-barotropic equations govern the dynamics of a semi-infinite gas layer, which is bounded below by a material surface. The bounding surface deforms according to the local temperature on the surface. In this work, the material surface is an isentropic surface, $\theta_{0}=$ constant. The governing equations read (Salby 1989):

$$
\begin{aligned}
\frac{D \mathbf{v}}{D t} & =-\nabla\left[\mathcal{H}+\mathcal{H}_{B}\right]-f \mathbf{k} \times \mathbf{v}+\mathcal{D}_{\mathbf{v}} \\
\frac{D \mathcal{H}}{D t} & =-\kappa \mathcal{H} \nabla \cdot \mathbf{v}+\mathcal{D}_{\mathcal{H}}
\end{aligned}
$$


where

$$
\begin{gathered}
\frac{D}{D t}=\frac{\partial}{\partial t}+\mathbf{v} \cdot \nabla, \\
\mathcal{H}=\frac{\theta_{0}}{\mathcal{A}_{0} \Gamma}\left(\frac{p_{0}}{p_{\text {ref }}}\right)^{\kappa},
\end{gathered}
$$

and

$$
\mathcal{H}_{B}=\frac{z_{0}}{\mathcal{A}_{0}}
$$

The boundary condition,

$$
\frac{D \theta_{0}}{D t}=0
$$

is trivially satisfied in the adiabatic case. In the diabatic case, this equation with forcing terms becomes a formal addition to the set of equivalent-barotropic equations. In Eq. (5), the dependent variables are the barotropic transformations of the original baroclinic variables in Eq. (1). For example,

$$
\mathbf{v}(\mathbf{x}, t)=-\frac{1}{p_{0}} \int_{p_{0}(\mathbf{x}, t)}^{0} \mathbf{v}(\mathbf{x}, p, t) d p
$$

for the baroclinic velocity, $\mathbf{v}(\mathbf{x}, p, t)$. Other variable have been similarly transformed. An equivalentbarotropic structure function, $\mathcal{A}=\mathcal{A}(\theta(p))$, is defined such that the inverse transformation for $\mathbf{v}$ is

$$
\mathbf{v}(\mathbf{x}, p, t)=\mathcal{A}(\theta) \mathbf{v}(\mathbf{x}, t)
$$

with the normalization,

$$
\frac{1}{p_{0}} \int_{0}^{p_{0}(\mathbf{x}, t)} \mathcal{A} d p=1
$$

In Eqs. (7) and (8a), barotropic and baroclinic forms are both represented by the same variable, but that should not elicit confusion. The D's in Eqs. (5) represent dissipation, as before. The subscript " 0 " (as in $p_{0}$ or $\theta_{0}$, for example) refers to the value of the variable at the bottom bounding surface. Accordingly, $\mathcal{A}_{0}=\mathcal{A}\left(\theta_{0}\right)$ is a measure of the local baroclinicity (vertical shear, or lack of vertical alignment), at the bounding surface. For an equivalent-barotropic structure which decays (grows) vertically, $\mathcal{A}_{0}$ must be greater (less) than 1 to preserve the normalization condition. In stronglystratified atmospheric regions, in which the flow structures tend to be vertically aligned, $\mathcal{A}_{0}$ should be close to unity. We have varied $\mathcal{A}_{0}$ from 0.8 to 1.2 and find no qualitative changes in the results. In all the simulation presented in this paper, $\mathcal{A}_{0}=1$. In Eq. $(5 \mathrm{e}), z_{0}=z_{0}(\mathbf{x}, t)$ is the prescribed elevation of the bounding surface. Using the definition of $\theta$, we have:

$$
\mathcal{H}=\frac{T_{0}}{\mathcal{A}_{0} \Gamma}=\frac{H_{p 0}}{\mathcal{A}_{0} \kappa} .
$$

Thus, the thickness, $\mathcal{H}=\mathcal{H}(\mathbf{x}, t)$, is essentially the temperature at the bounding surface. It is closely related to the local pressure scale height evaluated at the bounding surface, $H_{p 0}=R T_{0} / g$, 
and defines the potential temperature scale height, $H_{\theta 0}=H_{p 0} / \kappa$. It can be readily seen from Eqs. (5) that prescribed heating forces the flow through the deflection of the bounding surface, which advects the temperature. The advected temperature in turn further drives the flow.

When $\kappa=1$, the set of Eqs. (5) is formally identical to the shallow-water equations, with bottom topography (cf., Pedlosky (1987)). From the point of view of shallow-water dynamics, $\kappa$ (which is always $<1$ ) primarily produces enhanced advection. As in the shallow-water equations, Eqs. (5) admit an important conservation law for the potential vorticity $q$ :

$$
\frac{D q}{D t}=\mathcal{D}_{q},
$$

where

$$
q(\mathbf{x}, t) \equiv\left[\frac{\zeta+f}{\mathcal{H}^{1 / \kappa}}\right]
$$

and $\mathcal{D}_{q}=\mathcal{D}_{q}\left(\mathcal{D}_{\mathbf{v}}, \mathcal{D}_{\mathcal{H}}\right)$ is potential vorticity dissipation. In the absence of dissipation, the conservation law becomes:

$$
\frac{D q}{D t}=0 \text {. }
$$

Eqs. (10) and (11) are the equivalent-barotropic generalization of the shallow-water potential vorticity conservation. According to Eq. (11), $q$ is materially conserved in the absence of dissipation and can therefore serve as a proper tracer of the flow. In this work we will make frequent use of $q$ to visualize the flow. In observed atmospheres, $q$ is conserved very well, especially near the tropopause and above. Isentropic maps of $q$ have been used very effectively to gain much understanding in atmospheric dynamics studies of the Earth (cf., Hoskins et al. (1985)). Indeed, one of the significant advantages of our approach is our ability to conserve this crucial quantity to a high degree. This is achieved through the high resolution of our calculations and the accuracy of the employed numerical method, which we now briefly describe.

\subsubsection{Numerical Solutions}

Eqs. (5) are solved numerically in full spherical geometry - with $\mathbf{x}=(\lambda, \phi)$, where $\lambda$ is the longitude and $\phi$ is the latitude. The equations are solved in the vorticity-divergence form-i.e., the curl and divergence of the Eqs. (5a), along with Eq. (5b). In this form, the field variables, $(\zeta, \delta, \mathcal{H})$ $=\left(\mathbf{k} \cdot \nabla \times \mathbf{v}, \nabla \cdot \mathbf{v}, \mathcal{H}\left(T_{0}\right)\right)$, are projected onto the Legendre basis via the Gauss-Legendre transform (Orszag 1970; Eliassen et al. 1970):

$$
\xi(\lambda, \phi, t)=\sum_{n=0}^{N=N(M)} \sum_{m=-N}^{M} \hat{\xi}_{n}^{m} P_{n}^{m}(\phi) e^{i m \lambda},
$$

where $\xi$ represents an arbitrary field variable, $P_{n}^{m}$ is the associated Legendre polynomial for total wavenumber $n$ and sectoral wavenumber $m$, and $N$ and $M$ are the total and sectoral truncations. 
When $N=M$, the truncation mask is a triangle in spectral space, and the resolution of a calculation is referred to as "T106", for example, if $N=106$. In physical space, this truncation corresponds to a minimum of (longitude, latitude) $=(3 N+2=320,(3 N+2) / 2=160)$ grid points (Orszag 1970). In most cases, a spectral calculation is computationally much more accurate (though less efficient), per degree of freedom, than a finite difference calculation (Canuto et al. 1988; Haltiner and Williams 1980). The high resolution and accuracy achieved in the spectral algorithm is crucial for allowing small-scale structures and turbulence to evolve without suffering much dissipation. A small hyperviscosity, of the form $(-1)^{\chi+1} \nu \nabla^{2 \chi}$ for $\chi=\{1,2,3,4,8\}$, is included in Eq. (5) to stabilize the numerical integration while extending the turbulent inertial range. Details on the use and effects of hyperviscosity can be found in Cho and Polvani (1996a). As for the time-integration, the second-order accurate leapfrog scheme is used. We have thoroughly explored the numerical parameter-space associated with the present work and identified the region in which our results do not depend qualitatively on the precise choice of numerical parameter values.

Throughout this paper, simulations described are initialized with a random turbulent flow. This initialization crudely represents the effect of baroclinic eddies (arising from free and sloping convection and shear instability, for example) and its use is nearly universal in turbulence simulation studies. The random vorticity field is characterized by a narrow band in spectral space with a specified mean amplitude that corresponds to a global root mean square velocity, $\bar{U} \cdot{ }^{10}$ The divergence field is set to be uniformly zero initially. Given these fields, the remaining variable, $\mathcal{H}$, is obtained by requiring that the initial flow be nonlinearly balanced-i.e., satisfy the divergence of Eq. (5b) in the limit $\partial / \partial t \rightarrow 0$. This procedure allows the turbulent calculation to proceed over long times without "blowing up". A detailed discussion of the initialization procedure is also given in Cho and Polvani (1996a). We have verified that the results are insensitive to the location of the initial distribution's peak in spectral space, as long as the location is much smaller than the scale defined by $L_{\beta}$ (Rhines scale). The mean amplitude of the initial perturbations is a free parameter of the model, which we express here in terms of $\bar{U}$.

The equivalent-barotropic model admits the following parameters: $\mathcal{A}_{0}, \kappa, R, \Omega, g, R_{p}, \bar{U}$, and $\bar{T}_{0}$. Here, $\bar{T}_{0}$ is the global mean $T_{0}$. Note that three parameters appear in the equivalent-barotropic model in addition to the ones that are present in the shallow-water model. They arise from the more explicit representations of the physics in the equivalent-barotropic model. The added "cost" of the extra variables is minimal, however, since nearly all of the needed parameters can be reasonably constrained by observations or plausible scaling analysis. If Jupiter is used as a paradigm giant planet to constrain $R$ and $\Omega$ (for unsynchronized extrasolar giant planets), $\bar{U}$ becomes the most uncertain of the parameters. For Jupiter, $R$ is $3779 \mathrm{~J} \mathrm{Kg}^{-1} \mathrm{~K}^{-1}$, using $\kappa=2 / 7$. We have found that a $\kappa$ value of $2 / 5$, appropriate for monatomic gases, also does not qualitatively change the results. In addition, $\bar{U} \approx 70 \mathrm{~m} \mathrm{~s}^{-1}$ for Jupiter. A conservative estimate of $\bar{U}$ for current extrasolar giant

\footnotetext{
${ }^{10}$ Note for reference that $\bar{U}$ in the upper tropospheres of the Solar System planets is between $\sim 30 \mathrm{~m} \mathrm{~s}{ }^{-1}$ and $\sim 400 \mathrm{~m} \mathrm{~s}^{-1}$.
} 
planets is a somewhat-uncomfortable wide range, from $\sim 10^{2} \mathrm{~m} \mathrm{~s}^{-1}$ to $\sim 10^{3} \mathrm{~m} \mathrm{~s}^{-1}$. While not stultifying, the uncertainty is not insignificant. As we shall show, values in the lower range can lead to identifiably different circulation pattern than those in the upper range. As yet, we are unable to definitively conclude on the exact circulation pattern without further observational constraint. Currently, there is no way to obtain $\bar{U}$ from first principles. Scaling analysis does suggest, however, that $\bar{U}$ may be more likely to fall on the lower side of the range (Cho, in preparation).

\subsection{Thermal Forcing}

Two points are important to note when addressing thermal forcing on extrasolar giant planets: i) in general, it is not the direct stellar irradiation but the net flux (i.e. that which is non-linearly modified by the circulation) that forces the temperature tendency (Eq. (1d)) and balances the flow; and, $i$ i) because some of the stellar irradiation will be rejected by the atmospheric gases and clouds, the radiative equilibrium temperature distribution is not known with certainty for extrasolar giant planets at the present time. Further observations are required. Note that $i i)$ necessarily implies that the strength and distribution of forcing in $i$ ) is unknown for extrasolar giant planets and must be treated as a parameter.

For the Solar System giant planets, for which good flux observations exist, it is reasonable to model their atmospheres as layers of uniform thickness (temperature). This is because the emergent flux (which is as much as, or larger than, the insolation) is nearly uniform on them and $\tau_{r} \gg \tau_{a}$. Indeed, the flux-associated equator-pole temperature gradient on Jupiter is only $\sim 3 \mathrm{~K}$ at all longitudes. This suggests that thermodynamics may be effectively decoupled from the dynamics, as has been done in Cho and Polvani (1996b). Such a prescription is also probably appropriate for many unsynchronized extrasolar giant planets, which may behave plausibly like a "warm Jupiter". However, it is not likely to be as valid for the extremely close-in giant planets, given the much smaller $\tau_{r}$ than for Jupiter and the expected high radiative-equilibrium temperature gradients. On these planets, the temperature at the substellar point should be much closer to the equilibrium temperature, compared with the polar-and if synchronized, antistellar-regions. In all cases, an estimate of the radiative-equilibrium temperature distribution is required to model the flow properly.

For synchronized planets, in the absence of circulation, the radiative equilibrium temperature on the day side is modeled as

$$
T_{\mathrm{eq}}(\lambda, \phi)=T_{*}\left(\frac{R_{*}}{D}\right)^{1 / 2}\left(1-A_{\lambda \phi}\right)^{1 / 4}(\cos \lambda \cos \phi)^{1 / 4},
$$

where $T_{*}$ and $R_{*}$ are the parent star's effective temperature and radius, respectively. Here, $D$ is the distance of the planet from the parent star; and, $A_{\lambda \phi}$ is the local value of the albedo, a function of the location on the day side. This definition differs from the standard, "averaged" one, often 
found in the literature:

$$
\bar{T}_{\mathrm{eq}}=T_{*}\left(\frac{R_{*}}{2 D}\right)^{1 / 2}[\hat{f}(1-A)]^{1 / 4},
$$

where $A$ is the geometric albedo (an averaged value over the entire day side) and $\hat{f}$ is a parameter that describes whether the stellar flux is re-emitted by the entire planet $(=1)$ or only the day side (=2). Note that because the albedo is a function of temperature and space (via cloud formation, for example), the radiative equilibrium temperature field itself is not really well defined - even in the absence of motion. However, these two definitions allows us to very roughly bracket the range of forcing amplitude that needs to be explored.

Prior to synchronization, the outgoing flux should be more "homogeneously distributed" over the planet's surface. There is no orography or topography to make the diabatic heating zonally asymmetric on a gaseous giant planet. The temperature resulting from a planet which emits homogeneously is given by $\bar{T}_{\text {eq }}$ in Eq. (14) with $\hat{f}=1$. After reaching synchronization, however, the incoming stellar flux distribution is not zonally-symmetric and the equilibrium temperature associated with this influx is not uniform. The maximum radiative equilibrium temperature is then given by $T_{\text {eq }}(\lambda=0, \phi=0)$ in Eq. (13) at the substellar point with the temperature profile decreasing as $(\cos \lambda \cos \phi)^{1 / 4}$ away from the point. Statically, the thermal forcing resulting from synchronization can therefore be modeled in first approximation as a permanent temperature perturbation superimposed on a layer of nearly uniform temperature, $\bar{T}_{\text {eq }}$. If the differences in local vs. global albedo values are ignored (i.e., $A=A_{\lambda \phi}$ assumed) for the sake of argument, Eqs. (13) and (14) indicate that the amplitude of thermal forcing at the substellar point could be as large as $\sim 40 \%$ in excess of the uniform temperature value, $\bar{T}_{\text {eq }}$ (obtained for $\hat{f}=1$ ). Given the above-mentioned uncertainties in the definition of radiative equilibrium itself, we will consider amplitudes of thermal forcing reaching up to $20-40 \%$ in our circulation models.

On the night side, absent any circulation, atmospheric temperature should be approximately the effective temperature determined by the internal luminosity. The internal luminosity is powered by the slow gravitational contraction and possible helium rainout in the planet $^{11}$ :

$$
T_{\text {eff }} \sim 100 \mathrm{~K}\left(\frac{L_{\text {int }}}{L_{\text {int,Jup }}}\right)^{1 / 4}\left(\frac{R_{p}}{10^{8} \mathrm{~m}}\right)^{-1 / 2},
$$

where $L_{\text {int,Jup }} \sim 8.7 \times 10^{-10} \mathrm{~J} \mathrm{~s}^{-1}$ is the estimated internal luminosity of Jupiter. With the observed temperature at the substellar point of HD $209458 \mathrm{~b}$ possibly as high as $~ 1700 \mathrm{~K}$ (cf., Deming et al. (2005); Seager et al. (2005)), the temperature difference between the substellar and antistellar points on this extrasolar giant planet could be as high as $\sim 1600 \mathrm{~K}$ in the absence of motion. Obviously such an enormous temperature difference would be quickly, and violently, neutralized by atmospheric motion as long as $\tau_{a}<\tau_{r}$. It is crucial to note that, if $\Omega \neq 0$, the readjusted flow state will not be that which merely balances the above temperature gradient but also one which is

\footnotetext{
${ }^{11}$ Other mechanisms may be at play as well (cf., Guillot et al. (2006)).
} 
very strongly modified by the planetary rotation. This is a fundamental property of rotating fluids (Gill 1982). Note also that $\tau_{r}$ is locally a nonlinear function of $T$ and of the motion. Venus, which has a remarkably homogeneous temperature distribution despite its extremely slow longitudinal variation of insolation, may serve as an example of such a post-adjusted distribution. Incidentally, during such an readjustment phase, the flow state may not be unlike the turbulent initial condition used in our simulations, since small-scale gravity waves and turbulence will be generated during the process.

The salient point here is that a reasonable response to irradiation is to make the atmospheric layer thicker (due to the warmer radiative equilibrium temperature) on the day side than on the night side, as dictated by Eq. (3). Accordingly, in this work, the flow is forced with heating by appropriately "puffing up" the atmospheric layer on the heated side while maintaining a global equilibrium temperature $\bar{T}_{0}\left(=\bar{T}_{\text {eq }}\right)$. This is accomplished by introducing a permanent deflection of the bounding material surface through a specified $z_{0}(\mathbf{x})$ in Eq. (5e). In most runs, the deflection is "grown" after the basic flow pattern is established with a characteristic $e$-folding time (usually $\sim 5 \tau$, where $\tau \equiv 2 \pi / \Omega$ is the rotation period) in order to minimize introducing a large unbalanced component (small-scale fast modes) in the flow. A sudden "turning-on" of the forcing introduces a large amount of fast modes that are in general insignificant for meteorology and are filtered out in numerical weather predictions (Haltiner and Williams 1980). No heat (thickness) is added overall, but merely redistributed by the flow. Generally, this is most appropriate when $\tau_{r} \gg \tau_{a}$ or when $\dot{q}_{\text {net }} \rightarrow 0$ (Eq. (1d)). Here, we are mainly concerned with the horizontally advected component of the temperature, neglecting exchanges with regions above and below the modeled layer. However, we stress that, because of the way in which the bounding surface is deflected, our forcing procedure is also useful in the presence of strong irradiation and very short $\tau_{r} \rightarrow 0$.

The fluid layer is bulged on the day side according to:

$$
\mathcal{H}(\lambda, \phi)=\overline{\mathcal{H}}+\mathcal{H}^{\prime} \cos \phi \cos \lambda
$$

and

$$
z_{0}(\lambda, \phi)=\bar{z}_{0}-z_{0}^{\prime} \cos \phi \cos \lambda
$$

where $\mathcal{H}^{\prime}, z_{0}^{\prime}>0$ are the constant, maximum perturbation amplitudes of forcing at the substellar point above the reference levels, $\overline{\mathcal{H}}$ and $\overline{z_{0}}$. $\mathcal{H}^{\prime}$ is set to ensure that the flow is down-gradient of the temperature distribution at $\tau=0$. Without loss of generality, we set $\overline{z_{0}}=0$, since it can be subsumed into $\overline{\mathcal{H}}$. The same perturbation amplitude, but with opposite sign, is applied to the layer on the night side so that the forcing integrated over the entire planet surface $S$ is zero and

$$
\frac{1}{4 \pi} \int_{S} \mathcal{H}(\lambda, \phi) d \phi d \lambda=\overline{\mathcal{H}}
$$

We have also performed simulations in which the day side merges smoothly into a flat night side and found that the results do not change qualitatively. According to the argument outlined above, if we define

$$
\eta \equiv \frac{\mathcal{H}^{\prime}+z_{0}^{\prime}}{\overline{\mathcal{H}}}
$$


we are interested in $\eta \leq 0.4$. Since the actual value of $\eta$ may be different for different extrasolar giant planets and different altitudes, we vary its value from 0 to 0.4 in our simulations.

Note that the deflection approach cannot be used in the much lower levels of extrasolar giant planets since the vertical entropy gradient becomes very shallow in the equatorial region and a $\theta$-surface cannot be practically defined there. ${ }^{12}$ The bulging associated with heating can in fact be represented either indirectly by lowering the height of the lower bounding surface (thus producing a "surface deficit") or directly by adding mass (thus increasing $\mathcal{H}$ ). Similarly, the reduced thickness of the fluid layer on the night side (due to cooling) can be represented by directly removing mass, or by increasing the height of the lower bounding surface. This is just a restatement of the hypsometric relation, Eq. (3). The direct method is "diabatic" and the indirect method is "adiabatic". We have investigated both methods but present only the adiabatic case in this paper. Extensive study of the diabatic case is presented elsewhere. Our simulations also indicate that the key results on the developed circulation do not depend qualitatively on the precise functional form of the differential thermal forcing - e.g., Gaussian or " $\cos ^{1 / 4}$ " dependence, as opposed to the simple "cosine" dependence of Eq. (16).

Because the bulge associated with thermal heating can introduce a large unbalanced component in the flow, as described above, it can quickly lead to a numerical instability. Recall that the pre-existing part of the flow is nonlinearly balanced. Unless the flow is "stabilized" with draconian dissipative measures, which are unphysical, a gentle "ramp up" to the equilibrium profile is necessary. Even in this case, the destabilizing effect of imbalance when the amplitude is large cannot be completely avoided. We have tested both instantaneous and gradual forcing schemes. In the instantaneous version, the fluid is fully bulged right at the beginning of the simulation. In the gradual version, forcing is slowly increased with time during the numerical simulation, up to the chosen $\eta$ value. Again, we have found that simulations with instantaneous or gradual forcings gave results that are qualitatively similar. Overall, we find that the developed flow is surprisingly robust under a wide variety of conditions. This is one of our principle conclusions from this study. We note that the gradual version of forcing is important because it is perhaps the closest representation in the context of our model of what physically happens to a zero eccentricity and inclination extrasolar giant planet atmosphere near the tropopause, as the planet slowly becomes synchronized. To clearly identify the consequences of synchronization on the atmospheric circulation regime, we first consider unsynchronized planets.

\footnotetext{
${ }^{12}$ Computationally, the large deflection required causes holes to develop in the model layer, leading to blow-up.
} 


\section{Unsynchronized Giant Planets}

\subsection{Solar System Giant Planets}

Given the limited amount of direct information on atmospheres for extrasolar giant planets, validation of theoretical models is critical. The current work is closely-related to the shallow-water equations studies by Cho and Polvani (1996a,b). Their findings are directly relevant and lends some credence to our work. Hence, we briefly summarize the salient features before presenting the equivalent-barotropic equations results. For shallow-water equations in spherical geometry, two dimensionless numbers define the dynamics for a fixed radius: the Rossby number, $R_{o} \equiv U /(f L)$, and the Froude number, $F_{r} \equiv U /(g H)^{1 / 2}$. These numbers arise from the ratio of terms in the momentum equation of the shallow-water equations. The numbers also appear in the equivalentbarotropic equations and control the dynamics in the same way. $R_{o}$ is a measure of relative vorticity compared to the planetary rotation (or, the rotation time compared to the advection time), and $F_{r}$ is the shallow-water analog of the Mach number in compressible flows. ${ }^{13} \mathrm{~A}$ convenient and important third dimensionless number can be constructed from the above two: the Burger number, $B_{u} \equiv F_{r}^{2} / R_{o}^{2}=L_{\mathcal{R}}{ }^{2} / L^{2}$. In rotating-stratified fluids, $L_{\mathcal{R}}$ acts as an $e$-folding length limiting vortex and jet interactions. In planetary applications, the $\beta$ (Rhines scale) effect is necessary, but not sufficient, to produce long-lasting banded structures; $L_{\mathcal{R}}$ must also be finite. That is, the fluid must have a free surface, or be 2D-compressible. Quantitatively, $L_{\mathcal{R}}$ must be $\lesssim R_{p} / 3$ for a banded structure to be stable. In their studies, Cho and Polvani (1996a,b) use the simplest form of shallowwater equations, without external forcing or topography, in order to clearly delineate the intrinsic flow evolution from that due to forcing. Only a delta-function force in time is used to stir the fluid at the beginning of the simulation. In the cases in which a simple, continuous Markovian random forcing is used, the physical-space evolution is strongly influenced by the forcing and vortices do not merge robustly (Cho et al. 1997).

Because of the close relationship, shallow-water equations results can be used to validate the current model. We do this by performing simulations of Solar System giant planet atmospheres using the adiabatic equivalent-barotropic equations. This not only gives confidence in the present code, it is also sets the context for the more complex diabatic calculations. In addition, direct comparisons with shallow-water equations simulations allow the effects of additional physical parameters in equivalent-barotropic equations simulations to be carefully assessed. Since the primitive equations require even greater number of parameters, rendering model results sometimes difficult to interpret clearly, equivalent-barotropic equations simulations provide a good benchmark for full primitive equations models (e.g., Showman and Guillot (2002) and Cooper and Showman (2005)). The use of shallow-water equations model to elucidate or verify primitive equations model results is a common procedure in atmospheric circulation studies. Moreover, present calculations of Solar

\footnotetext{
${ }^{13}$ In the shallow-water equations, surface gravity waves are admitted, not sound waves, since the fluid is assumed homogeneous and incompressible in 3-D.
} 
System giant planets are useful in themselves since there have never been simulations of Solar System giant planets using the equivalent-barotropic equations. The calculations also have value in that Solar System giant planets still serve as paradigmatic giant planets in many ways; there must surely be extrasolar giant planets which are Solar System giant planet-like in their atmospheric properties. Finally, we note that the equivalent-barotropic equations are preferable because they derive directly from the primitive equations, and certain parameters (e.g., $L_{\mathcal{R}}$ ) acquire a more physical, less arbitrary, interpretation than in the indirectly related shallow-water equations.

Figures 1-6 show examples of Solar System giant planet simulations from our study. The global planetary parameter values adopted for the simulations are listed in Table 1 . The values for HD $209458 \mathrm{~b}$ are also included in the Table for comparison. In the simulations, small finite eccentricities are ignored. Since it is known from observations that the emitted flux is fairly uniform over the entire surface on all of the Solar System giant planets, we do not include any differential thermal forcing in our simulations of these planets. The success of these calculations in reproducing the global circulation pattern of Solar System giant planets gives confidence in our approach to modeling Solar System giant planets, as well as unsynchronized extrasolar giant planets with small eccentricities.

Figures 1 and 2 show a typical evolution from one of our Jupiter simulations (Model S1 in Table 2). Contour maps of the potential vorticity $(q)$ field are presented. Recall that $q$ is a tracer of the fluid, hence a proper variable to plot. Positive values are contoured with full lines, and negative values are contoured with dashed lines. In Figure 1, the maps are in orthographic projection centered on $(\lambda, \phi)=(270,0)$ and gridded at 30-degree intervals. Note that with this projection the polar latitudes occupy a smaller area of the disk than the low latitudes. The resolution of the simulation is T170 - i.e., $N=170$ in Eq. (12) - and corresponds to a $512 \times 256$ longitude-latitude grid over the globe. This resolution is minimally comparable to a $1024 \times 512$ grid in a finite difference calculation because of greater intrinsic accuracy of the pseudospectral method. The duration of this run is 2000 planetary rotations (i.e., $\tau=2000$ ). We have also performed shorter duration runs at higher resolutions (T213 and T341) to verify convergence of the results and find that there is no qualitative difference in the evolution at the higher resolutions. However, a minimum of T63 $(192 \times 96)$ resolution is required to resolve the turbulence (i.e. growing vortices); note that T63 is above the horizontal resolution of the simulations in Cooper and Showman (2005). Time in unit of the planetary rotation periods is indicated in the upper left corner of each frame. The contour levels, 40 in all, are identical in each frame. In Figure 2, the maps are in polar stereographic views centered on the north pole. In this projection also, the polar region occupies a smaller area of the disk than the equatorial region. East is in the counter-clockwise direction, and $\lambda=0$ is at the 3 o'clock position.

At $\tau=0$, the atmosphere is stirred randomly and released with a background corresponding to the planetary vorticity $f$. By $\tau=84$, the formation of zonally elongated structures is clearly evident at low latitudes. These structures are formed by growing eddies, which radiate Rossby (planetary) waves after reaching the size of $L_{\beta}$ (see $\S 2$ ). Rossby waves are the undulations of $q$ 
about a latitude circle. Note that the wave propagation is strongest at the equator since the linear dispersion relation from Eq. (11) for the Rossby waves gives a longitudinal phase speed,

$$
c_{p}=-\frac{\beta}{n(n+1) / R_{p}^{2}+1 / L_{\mathcal{R}_{e}^{2}}^{2}} .
$$

Recall that $\beta \equiv R_{p}^{-1} d f / d \phi=2 \Omega \cos \phi / R_{p}$ and is a maximum at the equator. The length, $L_{\mathcal{R} e} \equiv\left(g H_{e}\right)^{1 / 2} / f$, is the "equivalent depth" Rossby deformation radius, where $H_{e}$ is the equivalent depth - an important parameter in atmospheric dynamics (Gill 1982), including in the theory of tides (Chapman and Lindzen 1970). In the equivalent-barotropic model, $H_{e}=H_{p 0} / \mathcal{A}_{0}$, a weighted pressure scale height evaluated at the lower bounding surface. Note also that $L_{\mathcal{R} e}$ is largest at the equator and smallest at the pole, giving a small interaction length for the vortices in the polar region (see $\tau=423$ frame in Figure 2, for example). The small interaction length significantly reduces motion and mergers in that region. From our simulations, we expect the polar region at the cloudtop level of Jupiter to contain spots, rather than bands. And, in fact, bands are not observed above about 60 degrees latitude on Jupiter.

Maps of $q$ can be effectively used to study atmospheric circulation. Such maps constitute a standard tool for studying the circulation on the Earth (Hoskins et al. 1985). They have frequently provided insights to important processes which are otherwise difficult to obtain. Since $q=q(\mathbf{v})$, under certain "balanced" conditions (e.g., pressure gradient force balanced by the Coriolis force ${ }^{14}$ ), $q$ can be inverted to obtain the wind field, $\mathbf{v}=\mathbf{v}(q)$. That is, the wind information is contained in $q$. In spectral space, $q$ can be seen to have a broad distribution. This demands a high resolution for accurate representation, both observationally and numerically. Loss of $q$ due to under-resolving, therefore, causes loss of pertinent information. Indeed, the ability to retain $q$ is one strong justification for using the equivalent-barotropic model and also an important advantage of our model compared with models using the finite difference method.

Finely resolved $q$ features can be used to identify the presence of atmospheric flow structures (e.g., vortices and jets), as well as flows around the structures, to exquisite detail (cf., Cho et al. (2001), and references therein). For identifying jets, consider a simple 2-D zonal flow: $u=u(\phi)$ and $v=0$ with $u, v \in \Re^{2}$. If incompressible (non-divergent) in $2-\mathrm{D}, \mathcal{H}$ is constant. Then, $D q / D t=0$ with $q=(\zeta+f)$, and $\mathcal{H}$ plays no dynamical role. Using the definition of $\zeta(\equiv \mathbf{k} \cdot \nabla \times \mathbf{v})$, we have $\zeta=-R_{p}^{-1} \partial u / \partial \phi$. Note that, if $R_{o} \ll 1$ and $\zeta \sim U / L$, then $\zeta \ll f$. Therefore, for rapidlyrotating planets (such as Jupiter), the Coriolis parameter $f$ dominates over the relative vorticity $\zeta$, especially at high latitudes. In this case, $q$ is mostly positive (negative) in the northern (southern) hemisphere, as can readily be seen in Figure 1. Recall that $f=2 \Omega \sin \phi$ and hence changes sign when crossing the equator. The gradient of $q$ in the meridional direction is then

$$
\frac{\partial q}{\partial \mu}=\frac{\partial f}{\partial \mu}-R_{P}^{-1} \frac{\partial^{2} U}{\partial \mu^{2}}
$$

\footnotetext{
${ }^{14}$ This condition is known as geostrophic balance (Holton 1992) and applies to Solar System giant planets, as well as the Earth, to a good degree away from the equator.
} 
where $U=u \cos \phi$ and $\mu=\sin \phi$. On the plane tangent to the planetary surface, this is just

$$
\frac{\partial q}{\partial y}=\beta-\frac{\partial^{2} u}{\partial y^{2}},
$$

where $\beta=\partial f / \partial y$ and $y$ is the northward direction. According to Eqs. (20) and (21), jets may be identified by their curvatures. For example, in Eq. (21), an eastward jet corresponds to $\partial^{2} u / \partial y^{2}<0$ while a westward jet corresponds to $\partial^{2} u / \partial y^{2}>0$. Consequently, for a given value of $\beta$ (which is always positive), a large (positive) value of $q$ gradient indicates an eastward jet in the flow while a small (negative) gradient of $q$ indicates a westward jet. Thus, an atmosphere containing alternating eastward and westward jets show a corresponding alternating tightening and relaxing of $q$-contours in the meridional direction. The correspondence can be seen in Figures 2 and 3. The absolute value of $\partial q / \partial y$ ( or $\partial q / \partial \phi)$ is a direct measure of the sharpness of the jet, eastward or westward.

Figure 3 depicts the quasi-steady jets from the Jupiter simulation presented in Figures 1 and 2 (Model S1). It shows a zonally-averaged (eastward) wind profile as a function of latitude, [u], where

$$
[u(\phi)]=\frac{1}{2 \pi} \int_{0}^{2 \pi} u(\phi, \lambda) d \lambda .
$$

The jets are the peaks in the wind profile. The time of the simulation is $\tau=300$, well after the profile has emerged from the initial stirring. The profile is steady and nearly identical even at $\tau=2000$. In the figure, there are about half dozen zonal jets in each hemisphere with amplitude $\sim 25 \mathrm{~m} \mathrm{~s}^{-1}$. There is also a very strong equatorial jet of amplitude $\sim-125 \mathrm{~m} \mathrm{~s}^{-1}$. Qualitatively, the number, widths, and absolute magnitudes of jets are all similar to those observed on Jupiter. As in the previous shallow-water studies of Jupiter (Cho and Polvani 1996b), however, the sign of the equatorial jet is opposite to that observed on Jupiter, as well as on Saturn (not shown). The retrograde (westward) equatorial jet in the simulation is due to the strong presence of Rossby waves at the equator, as discussed above. Clearly, an additional driving mechanism is also required in the equivalent-barotropic equations model to capture the proper sign of the equatorial jet, as in the shallow-water equations model. This is not unexpected given the formal similarity between the equivalent-barotropic equations and the shallow-water equations. Later, in $\S 5$, we return to the issue of prograde (eastward) equatorial jet - currently an unsolved problem in planetary and stellar atmospheric dynamics. For now, we simply note that the model does capture the proper sign of equatorial jets on Uranus and Neptune, as we shall show shortly. Evidently, some mechanism not included in the simplest version of the equivalent-barotropic equations is required for Jupiter (cf., Heimpel et al. (2005)) and Saturn but not for Uranus and Neptune.

In Figure 4, we present a direct comparison of the equivalent-barotropic and shallow-water models. We do this to demonstrate the validity of our model. When $\kappa=1$, the adiabatic equivalentbarotropic equations formally reduce to the shallow-water equations, with $\mathcal{A}_{0}$ set to unity. ${ }^{15}$ In

\footnotetext{
${ }^{15}$ We remind the reader that vortex columns cannot tilt in the shallow-water model. So, a unit $\mathcal{A}_{0}$ is physically consistent.
} 
this case, $H_{p 0}$ becomes the layer thickness in the shallow-water model (Eq. (9)). Note that, while formally equivalent, the "shallow-water limit" of equivalent-barotropic equations does not carry a physical interpretation. This is because physically $\kappa=1$ corresponds to $c_{v}=0$ and $\gamma \rightarrow \infty$, where $c_{v}$ is the specific heat at constant volume and $\gamma=c_{p} / c_{v}$ (recall that $\kappa=R / C_{p}$ ). In the figure, two runs are presented: on the left (a) is a frame from the equivalent-barotropic calculation and on the right (b) is a frame from the shallow-water calculation (Models J1 and J2 in Table 2, respectively). In the two runs, all parameters are identical, except the equivalent-barotropic equations model calculation on the left has $\kappa=\mathcal{A}_{0}=1$. The resolution of the runs is T106. The projections of the maps are polar stereographic, as in Figure 2. The time of the frame is at $\tau=179$, long after the quasi-steady state has been reached. In these two runs, the initial spectrum is peaked at a lower wavenumber (larger scale) than in the run presented in Figures 1 and 2. As a consequence, the steady state is reached earlier in these runs, compared to the run in Figures 1 and 2. However, because both runs start with peaks at scales much smaller than the Rhines scale $L_{\beta}$, there is no qualitative difference in the final flow configuration: only the time when the equilibrium state is reached is different. Significantly, the results from the two models, (a) and (b), are practically identical. ${ }^{16}$ This verifies that flow features are truly robust (independent of the randomness of stirring, for example) and are reproducible by the new model.

Figure 5 shows three successive equatorial orthogonal (a) and polar stereographic (b) views of a typical Neptune simulation from our model (Model S4 in Table 2). In the figure, three time frames are shown to illustrate $i)$ the flow in the initial state $(\tau=0)$, ii) during transition to the quasi-steady equilibrium state $(\tau=33)$, and $i i i)$ the equilibrium state itself $(\tau=146)$. As in Figures 1 and 2, iso- $q$ contours are plotted with positive (negatives) values in full (dashed) lines. The initial spectral distribution is the same as in that of Figures 1 and 2, except $\bar{U}$ is larger (see Table 1). In this simulation, the structures dominated by scales around the wavenumber $n \sim 14$ at $\tau=0$ are more easily recognized than in the Jupiter case because of the larger $\bar{U}$ and smaller $f$ for Neptune. More importantly, because of the larger $\bar{U}$ and smaller $f$, the flow quickly evolves into a final configuration dominated by two strong circumpolar vortices (one centered on each pole) and a broad jet, associated with a well-homogenized $q$ region at low latitudes. In fact, only a few $q$ contours are seen all the way up to the high latitudes, until the boundaries of the polar vortices are reached. Such a $q=0$ low-latitude region was previously assumed in modeling the chaotic, wobbling motion of Neptune's Great Dark Spot with great success (Polvani et al. 1990). The strong polar vortex results from vigorous mergers, in which surrounding vortices are continuously "soaked up" before they are sheared away or dissipated. Dynamically, the strong interaction is expected given the large value of $L_{\mathcal{R}} / R_{p} \sim 1 / 6$ for Neptune, in contrast with $\sim 1 / 30$ for Jupiter (see Table 2). Note that the circumpolar vortices are cyclonic. Under geostrophic balance, the cyclonic vortex is associated with a cooler region than its surroundings.

In the case of Neptune as well, the steady-state configuration (already well in place after $\tau \sim 30$ )

\footnotetext{
${ }^{16}$ They of course cannot be exactly identical because of the initial random seeding and minor numerical differences.
} 
is stable over timescales of several thousand $\tau$. Significantly, as will be shown shortly, Neptune's jet profile is closer to that we obtain for close-in extrasolar giant planets than Jupiter, as reported in Cho et al. (2003). This marked difference mainly arises from the lower $\Omega$ values of close-in extrasolar giant planets compared to that of Jupiter. In fact, assuming synchronization, closein extrasolar giant planets rotate only moderately fast compared to Solar System giant planets. Additional differences between close-in extrasolar giant planets and Neptune also result from the intense irradiation experienced by the close-in extrasolar giant planets, as we shall see below ( 55 ).

But, first we quickly summarize our equivalent-barotropic equations results for the Solar System giant planets. Figure 6 shows the zonal wind profiles at late times obtained in our simulations of all four Solar System giant planets (Models S1-S4 in Table 2): Jupiter (a), Saturn (b), Uranus (c), and Neptune (d). Qualitatively, these profiles match well the observed profiles in terms of the overall number of bands and strengths of jets on each planet (see Cho \& Polvani 1996b for observed profiles). Generally, the four planets organize themselves into two discernible groups in terms of their general circulation patterns at the cloudtop levels. Jupiter and Saturn share similar flow patterns, with their multiple jets and narrow bands; and, Uranus and Neptune share similar flow patterns, with their few jets and broad bands.

In the current study, we have performed over 300 simulations in order to thoroughly explore the numerical and physical parameter-space spanned by the equivalent-barotropic equations system. Based on this extensive exploration, it appears that capturing the prograde rotation on Jupiter and Saturn requires physics beyond that included in the current, adiabatic equivalent-barotropic equations model. We give one example of a possible extension in $\S 5$. It is crucial to note that the lack of correspondence is not simply an issue of including the third dimension. One well-known mechanism in 3-D situation which does produce prograde rotation in simulations of the Earth (Suarez and Duffy 1992) is a zonally-asymmetric $(m=1$ and $m=2)$, large-amplitude forcing that is symmetric in the meridional direction. However, this mechanism does not apply to Jupiter (since it is not thought to be zonally asymmetrically heated) and cannot explain Saturn (since it is not meridionally symmetrically heated). In $\S 5$, we discuss the consequences of this forcing further for close-in extrasolar giant planets, where it may find some justification.

In summary, the results from our study illustrate several generic features of giant planet circulations modeled with the equivalent-barotropic equations. First, there is no qualitative difference between model results for equivalent-barotropic equations and shallow-water equations. Second, with the knowledge of a few global physical parameters, we are in a position to capture the gross properties of unsynchronized extrasolar giant planet atmospheric flows (several examples of possible unsynchronized extrasolar giant planet circulations are presented in the ensuing sections). Third, as in the previous shallow-water and equivalent-barotropic studies, the scales $L_{\beta}$ and $L_{\mathcal{R}}$ emerge as critical dynamical parameters for effecting realistic "predictions" of general circulations. Last, a knowledge of the strength and distribution of diabatic forcing (i.e., not just incident but also baroclinic, convective, latent heat, etc.) appears minimally needed to properly model the direction of the equatorial jet. 


\subsection{A Warm Jupiter}

Figure 7 illustrates our simulation of a "warm" Jupiter (Model U1 in Table 2). This may serve as an example of a Jupiter-like extrasolar giant planet which has migrated in some distance toward its host star but is not yet synchronized. At the same time, since the temperature increases with depth below the main cloud deck on Jupiter, it may heuristically serve as a representative flow at a deeper level in the Solar System planet. In this simulation, all the parameters are identical to that shown in Figures 1 and 2 (Model S1), except $\overline{\mathcal{H}}$ in this simulation is such that the global average temperature $\bar{T}_{0}=300 \mathrm{~K}$ (roughly a "Jupiter at $1 \mathrm{AU}$ "). Both stereographic (a) and orthographic (b) views are shown at $\tau=199$, well after the formation of steady bands and jets. This figure should be compared with the latter time frames of Figures 1 and 2 (Model S1).

In the early part of the evolution, the flow is very similar to the early evolution of Model S1. However, small differences begin to appear as the evolution proceeds: the banding at low to mid latitudes is weaker than that in Model S1 and even shows signs of slight erosion at times. The effect of the erosion can be seen in Figure 7, where the positive gradient of $q$ at low latitudes is weaker compared with those in Figures 1 and 2. The result is that bands generally appear more diffuse and there are one or two fewer jets in each hemisphere. The amplitude of the equatorial jet is reduced by more than $50 \%$ as well. This behavior is consistent with the findings in Cho and Polvani (1996a) for the shallow-water case and is due to the increase in $\overline{\mathcal{H}}$ leading to a larger $L_{\mathcal{R}}$ (Rossby deformation radius). The larger $L_{\mathcal{R}}$ acts as increased "stiffness", or reduced horizontal compressibility; this leads to stronger interaction between the structures in the fluid and the bands themselves undergo several "mergers", reminiscent of the vortices. Formally, the shallow-water equations reduce to 2-D, incompressible equations in the limit of $L_{\mathcal{R}} \rightarrow \infty$. Note that in the figure no mid-latitude anticyclonic spots survive and only a few vortices are present in the polar region, as a further consequence of the larger $L_{\mathcal{R}}$.

Given the behavior illustrated in Figure 7, we may draw several general conclusions. First, a much closer-in Jupiter may have a slightly different visual appearance than the present Jupiter at 5 AU. It will possess broad, diffuse bands with very few, possibly no, spots at the main cloud deck. Second, except for the equatorial jet, the overall jet structure is not changed very much, attesting to the strong control rotation has on the flow. The ratio, $L_{\mathcal{R}} / R_{p}$, is still $\ll 1 / 3$ in this simulation and a complete breakdown of the basic jet structure is not expected: an unrealistically large $\bar{T}_{0} \gtrsim 1.3 \times 10^{4} \mathrm{~K}$ is necessary for the breakdown. Finally, given that the situation can also be considered as crudely modeling a deeper level (one pressure scale height lower) in the current Jupiter, the flow is fairly vertically coherent (i.e., barotropic). There is not much difference in the

flow compared with that at the "higher level" (i.e., flow with $\bar{T}_{0}=130 \mathrm{~K}$ ), even in the absence of the explicit vertical coupling present in the baroclinic models. 


\subsection{Unsynchronized HD 209458 b}

Having established the singular importance of the rotation rate $\Omega$, by considering Jupiters with two different equilibrium temperatures (or orbital radii), we now consider the case of a "Jupiter" when its $\Omega$ is significantly reduced (e.g., by tidal interaction) but still not locked in 1:1 spin-orbit resonance. Such a planet would have a small semi-major axis, $a \lesssim 0.2 \mathrm{AU}$. This is an important case to study since it allows a clear delineation of the consequences of tidal locking on the atmospheric circulation. It is also relevant for the recently proposed scenario for HD 209458 b caught in a Cassini state (Winn and Holman 2005). As such, Figure 6 presents one of the key results of this study: atmospheric circulation on a planet with parameters representative of HD 209458 b (see Table 1) but without the explicit asymmetric forcing expected in the case of tidal synchronization.

In Figures 8 and 9, the flow evolution from Model U2 (see Table 2) is shown at the early stage (a) and at the equilibrated stage (b), respectively. Once the latter stage is reached, the flow state does not change in its essential character: the circulation is dominated by a single, coherent vortex at the pole and a sharp jet at its periphery, reminiscent of those on Neptune/Uranus rather than Jupiter/Saturn (compare with Figures 2 and 5). As in the simulations of Solar System giant planets, the initialization here is a random stirring, which is nonlinearly balanced and consistent with a specified $T_{0}$ and $\bar{U}$. In this simulation, $\bar{T}_{0}=1450 \mathrm{~K}$ and $\bar{U}=400 \mathrm{~m} \mathrm{~s}^{-1}$, plausible values for HD 209458 b. The actual values, particularly $\bar{U}$, are currently unknown. Note that these values give $L_{\mathcal{R}} / R_{p} \sim 1$ and $L_{\beta} / R_{p} \sim 1$, as shown in Table 2 .

Although clearly more similar to the circulation of Neptune/Uranus, there are some distinguishable differences. The polar vortex in Figure 9 is much larger than in the Neptune case. Also, the vortex is not centered on the pole, as it is on Neptune (see $\tau=146$ frame in Figure 5). Instead, it can be seen revolving around the pole on a timescale of about 2.7 planetary rotation periods in this run. In general, the timescale of the revolution around the pole depends on the strength and position of the polar jet (related to $\bar{U}$ ), since the polar vortex essentially moves with the jet (background zonal flow). In our extensive survey, we have found that the timescale of the revolution around the pole range from a few days to several tens of days. In addition, comparing the $\tau=146$ frame in Figure 5 with any of the frames in Figure 9, one can identify a marked reduction of Rossby wave amplitudes (undulations of $q$ lines about a latitude circle) at low-latitudes in the extrasolar planet case - although wave breaking (lateral overturning of a large-amplitude wave) occurs on both planets. Associated with this feature is the reduced mixing and homogenization at low-latitudes of this planet, compared with Neptune. All of these behaviors can be understood in terms of the larger $L_{\mathcal{R}}$ and smaller $\beta$ on the unsynchronized close-in extrasolar giant planet. Larger $L_{\mathcal{R}}$ means more robust mergers and a more dynamic final vortex. Smaller $\beta$ entails a weaker shearing environment for vortices at low latitudes and smaller wave amplitudes there.

An off-the-pole polar vortex, as in Figure 9, has several significant consequences for mixing of heat and vertical motion at high latitudes. If the vortex is cyclonic, as in the figure, the air in its interior is cooler than it's surroundings. Hence, a translating vortex carries with it a large mass of 
cold air around the pole. Moreover, as the vortex translates, it pulls up warm air from the lower latitudes ahead of it while pulling down cold air from higher latitudes behind it. This induces a poleward flux of heat and meridional mixing. The effect is accentuated by its meandering motion about a latitude circle. At the same time, the vortex cools in the net because it is generally warmer than the radiative equilibrium temperature (recall that very little stellar irradiation reaches the high latitudes). Hence, by the first law of thermodynamics, the potential temperature of the air inside the vortex decreases. But, since positive stability of a stratified region implies $\partial \theta / \partial z>0$, where $\theta(z)$ is the basic potential temperature, the cooling air must sink across $\theta$-surfaces to a lower valued surface; that is, there is a slow, down-welling motion inside the vortex (not modeled in this work). In addition, planetary waves further increase the downward drift by a hysteretic mechanism in diabatic conditions (Salby 1996). Later, in $\S 5$, we will show that planetary waves are induced by thermal forcing on extrasolar planets.

Figure 10a shows the quasi-steady jet profile from the simulation in Figures 8 and 9 (Model U2). In this simulation, in which $\bar{U}=400 \mathrm{~m} \mathrm{~s}^{-1}$ has been adopted, three broad jets form. The low number of jets is also a robust feature of our HD $209458 \mathrm{~b}$ simulations with day-night forcing (§5.1). While the magnitude and sign of individual jets vary from run to run, the number and root mean square speed of the jets mainly depend on $\bar{U}$, in rough agreement with $L_{\beta}$. Occasionally, when $\bar{U} \gtrsim 400 \mathrm{~m} \mathrm{~s}^{-1}$, a two-jet profile forms. The adopted $\bar{U}$ value is characteristic of Saturn, Uranus, and Neptune (see Table 1). On extrasolar giant planets, $\bar{U}$ can plausibly vary from $\sim 100 \mathrm{~m} \mathrm{~s}^{-1}$ to $\sim 1000 \mathrm{~m} \mathrm{~s}^{-1}$. A value many times greater than $\sim 1000 \mathrm{~m} \mathrm{~s}^{-1}$ is not likely to be realistic for large-scale flows on extrasolar giant planets since $c_{s} \lesssim 3000 \mathrm{~m} \mathrm{~s}^{-1}\left(\approx 2700 \mathrm{~m} \mathrm{~s}^{-1}\right.$ on HD 209458b). ${ }^{17}$ The two-jet configuration also forms in the special case when $\bar{U}=0$, as we will show in $\S 5.3$. At the low end of the range, when $\bar{U}=100 \mathrm{~m} \mathrm{~s}^{-1}$, a four or five jet profile can result, consistent with $L_{\beta}$. This case is illustrated in Figure 10b. Interestingly, the small $\bar{U}$ value is characteristic of the value at or above the cloudtops on Solar System planets with $a \lesssim 5$ AU. Hence, a simple scaling up of $\bar{U}$ with distance from the central star is not realistic and likely to be incorrect.

At this point we wish to remark on an issue pertaining to jets which requires clarification. There appears to be some confusion in the current literature concerning the universality of retrograde equatorial jets in one-layer turbulence calculations (cf., Cooper and Showman (2005)). We emphasize here that a retrograde equatorial jet is not a necessary outcome of all shallow-layer turbulence calculations. That outcome requires a small $L_{\mathcal{R}}$, as pointed out in Cho and Polvani (1996a). In the case of Solar System giant planets, $L_{\mathcal{R}}$ is indeed small for all the planets, and the equatorial jet is always retrograde in the absence of forcing. On extrasolar giant planets, $L_{\mathcal{R}}$ need not be small. In fact, $L_{\mathcal{R}}$ is generally not small for close-in extrasolar giant planets because they are much hotter and less-rapidly rotating than Solar System giant planets. Accordingly, the equatorial jet in Figure 10b is actually prograde. We have performed many simulations of HD $209458 \mathrm{~b}$ under a variety of conditions and find that what is robust is the adherence of the general flow pattern to

\footnotetext{
${ }^{17}$ This need not be so for small-scale waves, which may propagate with speeds close to or at $c_{s}$.
} 
$L_{\beta}$, not the direction of any particular zonal jet.

As already discussed, another dynamically relevant factor that is currently unknown is the radiative equilibrium temperature distribution on extrasolar giant planets. The distribution is related to the stellar irradiation but also depends in a complicated way on the composition, cloud and aerosol distribution, and the dynamics itself through the role it plays on the distribution of the radiatively active components. Figure 11 shows the simulation presented in Figure 6 (Model U2) at two different equilibrium temperatures: (a) $1800 \mathrm{~K}$ and (b) $800 \mathrm{~K}$ (Models U3 and U4, respectively). The range of the two temperatures is broad and is meant to roughly bracket $\bar{T}_{0}$ resulting from a variety of physical situations (e.g., distance, stellar flux, and albedo). With the same flux and albedo assumed in Model U2, the higher $T_{0}$ can also be applied to the case of an HD 209458 b-like planet which is $\sim 0.005$ AU closer to the host star than HD $209458 \mathrm{~b}$ at present $(a=0.045 \mathrm{AU})$. Similarly, the lower $T_{0}$ can be applied to an HD 209458 b-like planet that is $\sim 0.15$ AU further away. As can be seen in Figure 11, the higher $T_{0}$ leads to a more smoothly varying low- to mid-latitude $q$ distribution while the lower $T_{0}$ leads to much more planetary (Rossby) wave activity, along with a more centered polar vortex. In both cases, the basic three-jet profile is unchanged. However, interestingly, the higher $T_{0}$ value leads to displaced a polar vortex, which may potentially be of observational relevance (Cho et al. 2003).

\section{Synchronized Extrasolar Giant Planets}

\section{1. $\quad$ Effects of Zonally Asymmetric Heating}

From an atmospheric hydrodynamics point of view, HD $209458 \mathrm{~b}$ is particularly interesting in that both its radius and mass (hence its surface gravity) are accurately known and it has been the subject of most observational studies to date. The radius and mass information is now available for nine extrasolar giant planets and this number is expected to grow in the near future. As already noted, even if synchronization is invoked to pin down $\Omega$ and a "cloudless atmosphere" is considered to leave aside the complexities of radiative issues (clouds, albedo, vertical distribution of species, etc.), there is still no information available to constrain the amplitude of thermal forcing $\eta$ and the global wind speed $\bar{U}$. These parameters crucially control the flow dynamics through local changes of $L_{\mathcal{R}}$ and global changes of $L_{\beta}$. Eddy/wave amplitudes and propagations are affected as well. Experience with Solar System planets tells us that circulation and radiative transfer mutually interact in such a way as to modify the temperature structure away from the simple radiative and radiative-convective equilibrium. In this section, having considered a "HD $209458 \mathrm{~b}$ " in an unsynchronized state, we now isolate the effects of a specified value of $\eta$ on the flow. In $\S 5.2$, we present our findings of the effects of varying $\bar{U}$ on the flow and the horizontal temperature structure. We wish to focus on the robustness of plausible circulation patterns formed in realistic turbulent conditions.

As a first study of the forcing effect, we assume a simple day-night equilibrium temperature 
distribution, $z_{0}^{\prime} \propto(\cos \lambda \cos \phi)^{\varkappa}$, where $\varkappa$ is chosen from the set of $\{1 / 4,1,2\}$ for different runs. Note that a full 1-D radiative transfer calculation of HD $209458 \mathrm{~b}$ atmosphere applied over the planet suggests that this is a good range for the planet, with $\varkappa=1 / 4$ being more appropriate for the upper levels and $\varkappa=1$ being more appropriate for the lower levels in the stable layer of a close-in extrasolar giant planet (Cho et al., in preparation). There is no qualitative difference in our results due to a different choice of $\varkappa$ value adopted, however. The main result of our study of the forcing effect is as follows: in adiabatic, equivalent-barotropic, turbulent simulations, the global circulation is not qualitatively affected by the applied thermal forcing. That is, the general characteristics of global circulation (i.e., number of bands, strength of zonal winds, presence/absence of dominant polar vortices) are still well characterized by $L_{\mathcal{R}}$ and $L_{\beta}$ (or equivalently $R_{o}$ and $B_{u}$ ), irrespective of the value of $\eta$ adopted. This may appear surprising at first, but one of the key points of the present work is that the basic flow pattern is set by the dynamical parameters, $L_{\beta}, L_{\mathcal{R}}$, and $R_{p}$. Unless very strong diabatic forcing places the extrasolar planet into a special dynamical regime (see $\S 5.3$ ), forcing which merely deflects the isentropic surfaces (as opposed to causing large mass to cross the surfaces, as in overturning surfaces) cannot in itself produce major changes in the flow at this level of modeling. It can, of course, affect the temperature structure, as will be shown shortly.

The robustness of the basic flow is illustrated in Figure 12. The figure presents four T106 runs (Models H1, H2, H3, and H4 in Table 2), which are identical in all respects except for the forcing perturbation amplitude, $\bar{z}_{0}$. In the figure, $\bar{z}_{0}$ spans from 0 to $20 \%$ of the background temperature (i.e., $\eta=0$ to 0.2 ), corresponding to 0 to $\sim 300 \mathrm{~K} .{ }^{18}$ The latter is not a small deflection. We have also run cases in which the fractional deflections are even larger $(\eta=0.4)$, but the behavior is basically a more violent version of the $20 \%$ case. Frames at the same time $(\tau=95)$ from simulations with $\eta$ values of 0 (a), .02 (b), .04 (c), and .20 (d) are shown. As seen from the figure, the forcing primarily amplifies the mid-latitude jet. As $\eta$ increases, low latitude $q$ is homogenized, signifying strengthening of the retrograde equatorial jet $(\mathrm{a}-\mathrm{c})$. Concurrently, the $q$-gradient at midlatitude, hence the jet there, becomes sharper. The wave breaking activity in the mid-latitudes also diminishes, until the forcing is no longer small $(\mathrm{a}-\mathrm{c})$. In the large-amplitude case (d), the jet becomes strongly zonally asymmetric, becoming unstable, along with increased wave breaking activity in the mid-latitudes. When $\partial q / \partial \mu$ changes sign, due to the sharpening of the jet, the jet meets the necessary condition for barotropic instability and the unstable flow ensues (Charney and Stern 1962).

Figure 13 shows the effects of increased forcing amplitude $\eta$ on the temperature distribution, $T_{0}=T_{0}(\lambda, \phi)$, at dynamical equilibrium. The same runs in Figure 12 (Models H1, H2, H3, and H4) are shown with $\eta$ : (a) 0., (b) .02, (c) .04, and (d) .20. As can be seen, for a fixed $\bar{U}, T_{0}$ is strongly dependent on $\eta$. With no or low forcing, $T_{0}$ essentially tracks the flow, the polar vortex (a). As the forcing amplitude increases, however, competition between the flow-induced moving temperature pattern and the forcing-induced fixed temperature pattern develops: temperature anomalies on the

\footnotetext{
${ }^{18}$ Recall that the average background is represented by average thickness (mass) of the layer.
} 
whole either rotate quasi-steadily about the pole (b) or oscillate aperiodically about a point near the substellar point (c). When the amplitude is high, the forcing pattern dominates entirely and $T_{0}$ is essentially the imposed day-night distribution fixed on the substellar point (d). We emphasize here that the global circulation is not modified much, at least in the adiabatic case. Similar jet profiles and polar flow structures are present, even under strong forcing (compare Figure 13 with Figure 12): an asymmetric ${ }^{19}$ cyclone/anticyclone pair at high latitudes is always present (see Figures 12 and 15), but it may not be discernible in temperature maps if swamped by the applied thermal forcing. We note that when the same set of runs is repeated with larger $\bar{U}$, the same trends in temperature maps are exhibited at comparatively larger $\eta$ values, as expected from stronger motion-induced temperature patterns. Hence, thermal variability depends on $\bar{U}$, as well as $\eta$, as will be further demonstrated in the next subsection. This is one of our key results.

In summary, clearly, the entire spectrum of temperature distribution behavior is possiblefrom forcing being completely overwhelmed by the flow to forcing completely overwhelming the flow. Not unexpectedly, the critical $\eta$ value at which $T_{0}$ makes a transition to merely expressing the specified forcing depends on the given $\bar{U}$ value. The fact that the flow is fairly similar in all these cases indicates that the coupling between the flow and applied heating is relatively weak. Diabatic forcing appears to be required for a stronger coupling. The overall implication here is that, one cannot infer $\bar{U}$ and $\eta$ independently of each other from a temperature map, at least in adiabatic conditions.

\subsection{Global Mean Kinetic Energy Variation}

Figure 14 shows the dependence of the global circulation on $\bar{U}$. The figure presents four T106 runs (Models H1, H5, H6, and $\mathrm{H} 7$ in Table 2) that are identical in all respects, except for $\bar{U}$. In these runs, $\bar{U}$ spans from 100 to $1000 \mathrm{~m} \mathrm{~s}^{-1}$ from run to run. As noted already, the latter is not a small value: globally-speaking, the circulation is no longer in the quasi-geostrophic regime, when $\bar{U} \sim 1000 \mathrm{~m} \mathrm{~s}^{-1}$, since $R_{o} \sim 1$. Locally, of course, $R_{o}$ may be $\ll 1$, since the velocity varies over the planet. In the figure, simulations with $\bar{U}$ values of $100 \mathrm{~m} \mathrm{~s}^{-1}$ (a), $200 \mathrm{~m} \mathrm{~s}^{-1}$ (b), $400 \mathrm{~m} \mathrm{~s}^{-1}$ (c), and $1000 \mathrm{~m} \mathrm{~s}^{-1}(\mathrm{~d})$ are presented at the same time frame $(\tau=95)$. We have also run cases in which $\bar{U}$ is even larger $\left(2000 \mathrm{~m} \mathrm{~s}^{-1}\right)$, but in this case the behavior is similar to that of $(\mathrm{d})$, at early times. At later times, the flow field blows up, due to the large amount of surface gravity waves that naturally arise. $^{20}$ It can be seen clearly in Figure 14 that increasing $\bar{U}$ increases the size and strength of the polar vortex. In fact, the vortex is large enough in (d) to nearly cap the planet poleward of 60 degrees if it were centered at the pole. This results from the greater root mean square kinetic energy, $\frac{1}{2} \bar{U}^{2}$, of the initial eddies.

\footnotetext{
${ }^{19}$ The cyclone is much stronger than the anticyclone.

${ }^{20}$ The blowing up can be prevented, to a certain extent, if an unrealistically large amount of artificial dissipation is applied or, equivalently, if the resolution is very low.
} 
It may appear to the reader that, in Figure 14, planetary (Rossby) wave breaking activity abruptly terminates at high $\bar{U}$. However, in actuality, it increases. This is demonstrated in Figure $15 \mathrm{a}-\mathrm{c}$, in which the field presented in Figure 14d (reproduced in Figure 15a) is successively contoured at smaller range and intervals (Figure 15b-c); the number of contours is the same. The vortex is "chopped off" to reveal the surrounding flow. Clearly there is a large amount of structure outside the dominant vortex. In fact, the large amplitude of the planetary (Rossby) waves and their breaking has produced an anticyclone (Figure 15b). This anticyclonic vortex corresponds to a warm region compared to the interstitial region between the pair, giving rise to an associated slowly-rotating thermal dipole. This is remarkably similar to what happen on the Earth: in the Earth's stratosphere, upwardly-propagating planetary waves cause the stratospheric polar vortex to be displaced from the pole and induce the formation of an anticyclone, conserving $q$. The interstitial region itself is well mixed due to pronounced wave breaking, as on the Earth (cf., Andrews et al. (1987)).

Figure $15 \mathrm{~d}-\mathrm{e}$ shows the effect of increasing $\bar{U}$ on the zonal winds. The zonal wind profile is plotted for two simulations presented in Figure 14: (a) and (d)-Models H1 and H7, respectively. The figure clearly shows that, for fixed $\Omega$ and $R_{p}$, the number and width of the jets depend on $\bar{U}$ in good agreement with the $L_{\beta}$ estimate. Compare also this figure with Figure 10, in which asymmetric heating was not applied. From the comparison, one may draw two general conclusions: 1) forcing does not appear to have a distinguishable effect on the general structure of the zonal jets, their number and width; and 2) given the broad range of $\bar{U}$ values in the simulations of the two figures, the low number of zonal jets is a robust feature on HD 209458 b-like planets.

The $\bar{U}$ values can be shown to be intimately tied with the resulting temperature distribution, as shown in Figure 16. The same runs as in Figure 14 (Models H1, H5, H6, and H7) are shown with $\bar{U}$ values: (a) $100 \mathrm{~m} \mathrm{~s}^{-1}$, (b) $200 \mathrm{~m} \mathrm{~s}^{-1}$, (c) $400 \mathrm{~m} \mathrm{~s}^{-1}$, and (d) $1000 \mathrm{~m} \mathrm{~s}^{-1}$. As can be seen, for a fixed forcing amplitude $\eta, T_{0}$ is strongly dependent on $\bar{U}$. This is very similar to the behavior illustrated in Figure 13. With small $\bar{U}, T_{0}$ essentially expresses the day-night difference (a); that is, the flow is not energetic enough to advect temperature in significant amounts. As $\bar{U}$ increases, however, competition between the flow-induced temperature pattern and the forcing-induced temperature pattern develops: temperature anomalies on the whole either oscillate aperiodically about a point near the substellar point (b) or rotate quasi-steadily about the pole (c). When $\bar{U}$ is high, the flow dominates and $T_{0}$ essentially reflects the distribution dictated by the dominant vortices (d). Again, in each case, there is an asymmetric cyclone/anticyclone pair at high latitudes, independent of $\bar{U}$. When the same set of runs is repeated with larger $\eta$ values, the same trend is exhibited, with the transition to flow dominance occurring at larger $\bar{U}$ value. The only time a qualitative departure is obtained is in the special case when the simulation is started at a resting state and driven wholly by the applied day-night forcing (without any stirrings), the situation to which we now turn. 


\subsubsection{Quiescent Initial State: A Special Case}

At the current level of modeling, be it with the equivalent-barotropic equations or the full primitive equations, one of the ingredients necessary for accurate modeling and good understanding of extrasolar giant planet atmospheric circulation is the physics of baroclinic processes. To be more precise, what is important is the inclusion of the effects of the processes, not so much the actual processes themselves. This is because they are well below the modeled scales, as noted earlier. This is the idea of parameterization. In our usual model setup, the atmosphere is stirred in the beginning. The stirring is designed to crudely represent a generic form of baroclinic process: eddies and waves generated by small-scale convection or baroclinic instability, for example. Both of these generating mechanisms are expected to exist on extrasolar giant planets. The thermal forcing should be another source of strong eddies and waves, especially on close-in extrasolar giant planets. Once created, the eddies and waves transport and/or propagate crucial dynamical quantities, such as heat and wave-activity density ${ }^{21}$ (Andrews et al. 1987). For example, the action of waves greatly affect both the source and the target region (where the waves are dissipated) via interaction with the background flow (e.g., jets) present in the target region. This is one reason why we have emphasized resolution and laid particular stress on the jet profiles, and flow structures in general, in this study.

Recently, Showman and Guillot (2002) and Cooper and Showman (2005) have performed baroclinic simulations of close-in extrasolar giant planet atmospheres using the primitive equations. In these simulations, the atmosphere initially at rest is driven by day-night forcing applied in the energy equation. As in some simulations of "stripped-down" Earth, which employ the primitive equations and similar forcing (i.e., one that is low-order and meridionally-symmetric, but zonallyasymmetric), they obtain close-in extrasolar giant planet atmospheres which are superrotating. That is, the zonal wind at the equator is prograde: $[u(\phi=0)]>0$. In Earth simulations, the emergence of superrotation depends nonlinearly on the amplitude of the forcing, sometimes making an abrupt transition from subrotating to superrotating state at some critical amplitude (Suarez and Duffy 1992). At criticality, a bifurcation is observed, the flow exhibiting sometimes superrotation and sometimes not, for an identical set of parameters. In some cases, the superrotation appears to be in a stable equilibrium state, as the atmosphere remains superrotating even when the forcing is removed. Exactly how this peculiar behavior arises is currently not well-understood. Feedbacks between eddy momentum, angular momentum, Hadley cell, bottom friction, and vertical resolution appear to be at play. Certainly, vertical angular momentum exchanges between atmospheric layers and horizontal exchanges within a layer is required for superrotation.

In simulations of the Earth, superrotation has also been achieved, and investigated, through direct angular momentum forcing (Saravanan 1993). As with many phenomena admitted by the full primitive equations, superrotation of planetary atmospheres can also be studied using the

\footnotetext{
${ }^{21}$ For homogeneous fluids, wave-activity density is $\left[\frac{1}{2} \zeta^{\prime}\right] /[\partial q / \partial \mu]$, where $\zeta^{\prime}$ is the vorticity-eddy. It is sometimes also called (minus) pseudomomentum in the literature.
} 
equivalent-barotropic equations in a similar way. In this work (see also Shell and Held (2004)), an equatorial torque is applied to represent what is expected to happen to and within an individual layer in a (multi-layer) baroclinic atmosphere. This is done by adding a forcing term,

$$
\mathcal{F}=\tilde{\mathcal{F}} \cos ^{n} \phi
$$

to the zonal momentum part of Eq. (5a). Here, $n$ is an integer and is set to unity in the runs presented in this paper; we have checked that narrower forcing, given by $n=2$ or 4 , leads to qualitatively similar results. Note that the forcing is zonally-symmetric, even though the underlying baroclinic process modeled is zonally-asymmetric. As already discussed, the exact (and unknown) form of forcing need not be specified and is actually not sought: our aim here is an assessment of the evolution of an established superrotating equatorial jet and the nature of its interaction with eddies in an idealized setting. Accordingly, the forcing is assumed not to respond to the modeled layer itself (i.e., no back-reaction); we have in mind an "active" modeled layer that overlies a massive "abyssal" interior, which merely acts as a source of low angular momentum and friction (e.g., due to convective turbulence). One can think of this forcing as arising from some general zonally-asymmetric baroclinic process that produce eddy-momentum convergence at low latitude. A small amount of linear drag, of the form $-\alpha u$, is added to balance the forcing.

The resulting flow from Model Z1 in Table 2 is depicted in Figure 17. The resolution of the simulation is T106. The iso- $q$ contours in cylindrical projection, centered on the substellar point. Three time frames are shown: $\tau=\{35,40,100\}$ (Figure 17a-c, respectively). The corresponding zonal jet profiles in each time frames are shown as well. In this simulation, the force-dissipation balance is set so that the maximum wind is $\sim 1000 \mathrm{~m} \mathrm{~s}^{-1}$, similar to the simulation reported in Showman and Guillot (2002) at the 5.6-8.1 bar pressure level. A quiescent initial state is adopted, as in Showman and Guillot (2002) and Cooper and Showman (2005). In the figure, the first thing to note is that the flow is meridionally (north-south) symmetric, as in Showman and Guillot (2002). This is expected given the setup and indicates a complete dominance of the applied forcing. Particularly clearly visible in Figure 17a are the large Rossby waves that have been excited by the forcing. One can also clearly see the superrotation $([u(\phi=0)]>0)$ that is produced by the forcing in the corresponding jet profile. However, as the Rossby waves propagate meridionally, they interact with the background flow to drag the mid-latitude mean flow back (in the westward direction), simultaneously causing the equatorial mean flow (the source location) to speed up.

The behavior follows a well known acceleration/deceleration mechanism in atmospheric dynamics. The dissipation occurs in the region where the phase speed of the waves match the mean flow speed. Since Rossby waves propagate westward, the flow must be eastward for the mechanism to operate. Observe in Figure 17b that, where the wave has dissipated, large-scale eddies have formed. The flow is at this point very similar to that of Showman and Guillot (2002) (see their Figure 6). However, ultimately, the flow is not stable - at least in the angular momentum forced equivalent-barotropic system simulated here (Figure 17c). The resulting flow is very complex (compared to Showman and Guillot 2002), but the hemispheric symmetry still persists; the increased complexity is due to the higher resolution. One can see the strong influence of the propagating 
waves in Figure 17c, after which the jet profile does not change very much. Except for the equatorial jet, the mean flow is wholly westward from the time shortly before $\tau=100$. Clearly, the strength of superrotation depends on the pre-existing background flow, given the forcing amplitude.

Using the equivalent-barotropic model, we have also explored the behavior of superrotating flow states under various initial conditions. Our equilibrated-state findings are summarized in Figure 18, which present results from Models Z2, Z1, and Z3 in Table 2. The figure illustrates the importance of allowing eddies to be present in the flow field. The projections for $q$-field maps on the left column are as in Figure 17.

Figure 18a illustrates thermally forced $(\eta=0.4)$ flow without applied angular momentum forcing $(\tilde{\mathcal{F}}=0)$. The jet profile consists of a mid-latitude westward jet in each hemisphere, consistent with spreading of $q$ lines at mid-latitudes by the applied day-night forcing. The flow is not very "dynamic" in this case and does not change much over time. In contrast, with both angular momentum and day-night forcings applied, the flow, shown in Figure 18b, becomes more complex and dynamic, with the background mean flow interacting strongly with radiating planetary (Rossby) waves. Finally, we have in Figure 18c all three forcings (day-night, angular momentum, and initial random stirring) included in the simulation. Here, the flow is similar to the turbulent flow cases we have presented throughout this paper, with $\bar{U} \neq 0$. In this case, there are three broad jets. Because the $\bar{U}$ associated with the stirring is $400 \mathrm{~m} \mathrm{~s}^{-1}$, which is larger than the maximum jet amplitude without the stirring (see Figure 18b), the equatorial jet here is weakly retrograde. Hence, it appears that the strength of superrotation is sensitive to the vigor of eddies present. This suggests that the absence of vigorous eddies in the simulations of Showman and Guillot (2002) and Cooper and Showman (2005) may pose a dynamical limitation.

\section{Conclusion}

In this work, we have extensively explored global, turbulent, adiabatic atmospheric dynamics of extrasolar giant planets in circular orbits. Extrasolar giant planets with eccentricity $<0.05$ comprise a significant fraction of the currently known population. Several robust properties emerge from our set of simulations, which broadly confirm the results in Cho et al. (2003). First, there is a strong rotational control on the flow dynamics. This leads to atmospheric flows with a strong zonal component on extrasolar giant planets, independent of the thermal forcing amplitude. Hence, the velocity field is not one of a simple diverging flow away from the substellar point. This is so even for close-in extrasolar giant planets. This behavior is reminiscent of Uranus, which possesses strong zonal winds like the other Solar System giant planets even though it is heated at the pole. Second, in trying to understand the general circulation of extrasolar giant planet atmospheres, it is clearly useful to divide the task along the following two lines: i) understand the zonally-symmetric and zonally asymmetric circulation; and, ii) understand the tropical-subtropical and midlatitude-polar dynamics. Of course, each includes the interaction with the other two components. Third, in the adiabatic equivalent-barotropic equations model, it is possible to have a full range of global temper- 
ature distributions. They include rotating, oscillating, shifted, and fixed day-night distributions.

Not surprisingly, the spatiotemporal behavior exhibited by the temperature distribution depends on the amplitude of the thermal forcing, if the characteristic radiative time is not short compared to the characteristic advection time. When thermal forcing is strong, the day-night temperature difference is expected to be fixed. In effect, the temperature field decouples from the dynamics and can essentially be thought of as "thermal orography". However, the degree of decoupling depends on the strength of the background flow so that, if pre-existing zonal winds and eddies are strong, the temperature field is again enslaved to the flow field. Therefore, it appears from the adiabatic calculations performed in this work that it would be difficult to ascertain the characteristic flow speed independently of the radiative properties (e.g., albedo) - and vice versa. Note that because not as much irradiation is absorbed at higher altitudes (in the absence of special absorbers), the smaller amplitude forcing situations can be considered very roughly applicable to those regions. This would lead to a vertical shear in the temperature field and a baroclinic adjustment might be indicated. It would be interesting to study such adjustment processes when vertical communication between different layers is taken into account.

In general, the response of the atmospheric motion to both mechanical and radiative types of forcing is very complex and difficult to analyze from first principles. Modeling such complexity requires a range of approaches - from simple analytical calculations to full general circulation modeling. Different approaches are more successful with different pieces of the full problem, and they are all needed to make good progress. In this work, the equivalent-barotropic equations in isentropic coordinate have been used to focus on lateral dynamics near the top of the planet's convection zone. It is important to note that the isentropic layers above this region do not lie at constant geometric heights. The layers slant slightly downward from the substellar point to the poleward direction on extrasolar giant planets; on close-in extrasolar giant planets the layers slant downward toward the antistellar direction as well. On the other hand, the isentropic layers below the region (the top of the convection zone) slant upward by a very significant amount from the substellar point. In general, air parcels move both along and across these surfaces, transporting heat in both horizontal and vertical directions. We have taken advantage of the clear association of horizontal and vertical motions with adiabatic and diabatic heating, respectively, to perform equivalent-barotropic equations simulations in what amounts to " $1 \frac{1}{2}$-layers" (i.e., a layer with variable thickness).

Despite the vertical integration, the equivalent-barotropic equations support many of the phenomena and types of fluid motion supported by the primitive equations. This includes Rossby waves, gravity waves, balanced motions (e.g. geostrophic), adjustments, and barotropic instability. Important consequences of baroclinic processes, such as stirring by eddies spawned from baroclinic instability, can be well-resolved, allowing good physical representation via parameterization. The high resolution also allows mixing of $q$ and fine-scale tracers and turbulent cascades to small scales - all critical features in real atmospheres. Consequences of thermal forcing on eddies and mean-flow can be included through the deflection of lower boundary height. In addition, the equivalent-barotropic equations have a consistent set of conservation laws for mass, energy, angu- 
lar momentum, $q$, potential enstrophy $\left(\mathcal{H} q^{2}\right)$, and more exotic quantities like pseudomomentum (wave activity). In view of all these properties, we have carefully explored the dynamical behavior of the adiabatic equivalent-barotropic equations model in this work. In subsequent studies, we will consider more general physical situations important for understanding atmospheric dynamics on extrasolar giant planets. With the recent breakthrough detections of infrared emission from several close-in extrasolar giant planets, observational programs should be able to provide better constraints in the future on key atmospheric parameters, allowing more accurate assessments of the circulation and thermal structures on these planets.

\section{Acknowledgments}

This work was supported in part by NASA contracts NAG5-13478 and NNG06GF55G. JYKC thanks Ursula Wellen for helpful comments on the manuscript. 


\section{REFERENCES}

Andrews, D.L.T., Holton, J.R. \& Leovy, C. B., 1987, 'Middle Atmosphere Dynamics' (Academic Press, Orlando)

Batchelor, G.K. 1969, Phys. Fluids Suppl., 12 II, 233

Batchelor, G. 1977, 'Introduction to Fluid Dynamics' (Cambridge University Press, Cambridge)

Barman, T. S., Hauschildt, P. H. \& Allard, F. 2005, ApJ, 632, 1132

Burrows, A., Hubeny, I. \& Sudarsky, D. 2005, ApJ, 625, L135

Burrows, A., Sudarsky, D. \& Hubeny, I. 2006, ApJ, submitted (astro-ph/0607014)

Canuto, C., Hussaini, M.Y., Quarteroni, A. \& Zang, T.A. 1988, 'Spectral Methods in Fluid Dynamics' (Springer-Verlag, New York)

Chapman, S. \& Lindzen, R. 1970, 'Atmospheric Tides' (Reidel Publishing, Dortrecht)

Charbonneau, D., et al. 2005, ApJ, 626, 523

Charney, J. \& Stern, M. 1962, J. Atmos. Sci., 19, 159

Cho, J.Y-K. \& Polvani, L.M. 1996a, Phys. Fluids, 8, 1531

Cho, J.Y-K. \& Polvani, L.M. 1996b, Science, 273, 335

Cho, J.Y-K., Ingersoll, A.P., \& Polvani, L.M. 1997, BAAS, 29, 1017

Cho, J.Y-K., de la Torre Juárez, M., Ingersoll, A.P. and Dritschel, D.G. 2001, J. Geophys. Res., 106,5099

Cho, J.Y-K., Menou, K., Hansen, B.M.S. \& Seager, S. 2003, ApJ, 587, L117

Cooper, C.S. \& Showman, A.P. 2005, ApJ, 629, L45

Deming, D., Seager, S., Richardson, J. L., \& Harrington, J. 2005, Nature, 434, 740.

Deming, D. Harrington, J., Seager, S. \& Richardson, L. J. 2006, ApJ, 644, 560

Eliassen, E., Mechenhauer, B. \& Rasmussen, E. 1970, Rep. No. 2, Institut for Teoretisk Meteorologi, Kobenhavns Universitet, Denmark

Ferreira, R.N.\& Schubert, W.H., 1997, J. Atmos. Sci., 54, 261

Fortney, J. J., Marley, M. S., Lodders, K., Saumon, D. \& Freedman, R. 2005, ApJ, 627, L69

Gill, A. 1982, 'Atmosphere-Ocean Dynamics' (Academic Press, San Diego) 
Goldreich, P. \& Soter, S. 1966, Icarus, 5, 375

Guillot, T., Santos, N. C., Pont, F., Iro, N., Melo, C. \& Ribas, I. 2006, A\&A, 453, L21

Haltiner, G.J. \& Williams, R.T. 1980, 'Numerical Prediction and Dynamical Meteorology' (Second edition, John Wiley \& Sons, New York)

Heimpel, M, Aurnou, J. \& Wicht, J. 2005, Nature, 438, 193

Holton, J.R. 1992, 'Introduction to Dynamic Meteorology' (Academic Press, San Diego)

Hoskins, B.J., McIntyre, M.E. \& Robertson, A.W. 1985, Q.J.R. Met. Soc., 111, 877

Iro, N., Bézard, B. \& Guillot, T. 2005, A\&A, 436, 719

Juckes, M.N.\& McIntyre, M.E., 1987, Nature, 328, 590

Kolmolgorov, A.N. 1941, Dokl. Akad. Nauk. SSSR, 30, 9

Kolmolgorov, A.N. 1941, Dokl. Akad. Nauk. SSSR, 31, 538

Kolmolgorov, A.N. 1941, Dokl. Akad. Nauk. SSSR, 32, 16

Kraichnan, R.H. 1967, Phys. Fluids, 10, 1417

Laplace, P.S. 1778, Mem. Acad. R. Sci. Paris, 1775, 75

Leith, C.E. 1968, Phys. Fluids, 11, 671

Lubow, S. H., Tout, C. A. \& Livio, M. 1997, ApJ, 484, 866

Mac Low, M.-M. and Ingersoll, A. P. 1986, Icarus, 65, 353

McWilliams, J.C. 1984, J. Fluid Mech., 146, 21

Menou, K., Cho, J.Y-K., Seager, S. \& Hansen, B.M.S. 2003, ApJ, 587, L113

Ogilvie, G. I. \& Lin, D. N. C. 2004, ApJ, 610, 477

Orszag, S.A. 1970, J. Atmos. Sci., 1970, 27, 890

Pedlosky, J. 1987, 'Geophysical Fluid Dynamics' (2nd ed., Springer-Verlag, New york)

Polvani, L.M., Wisdom, J., DeJong, E. \& Ingersoll, A.P. 1990, Science, 249, 1393

Polvani, L.M., Waugh, D.W. \& Plumb, R.A., 1995, J. Atmos. Sci., 52, 1288

Rasio, F. A., Tout, C. A., Lubow, S. H. \& Livio, M. 1996, ApJ, 470, 1187

Rhines, P.B. 1975, J. Fluid Mech., 69, 417 
Salby, M.L. 1989, Tellus, 41A, 48

Salby, M.L., Garcia, R.R., O’Sullivan, D., Callaghan, P. \& Tribbia, D., 1990, J. Atmos. Sci., 47, 188

Salby, M.L. 1996, 'Fundamentals of Atmospheric Physics' (Academic Press, San Diego)

Saravanan, R., 1993, J. Atmos. Science, 50, 1211

Saumon, D. \& Guillot, T. 2004, ApJ, 609, 1170

Seager, S., Sasselov, D.D., 1998, ApJ, 502, L157

Seager, S., Richardson, L. J., Hansen, B. M. S., Menou, K., Cho, J. Y.-K. \& Deming, D. 2005, ApJ, 632, 1122

Shell, K. M. \& Held, I. M., 2004, J. Atmos. Sci., 61, 2928

Showman, A.P. \& Guillot, T. 2002, A\&A, 385, 166

Suarez, M. J. \& Duffy, D. G., 1992, J. Atmos. Sci., 49, 1541

Sudarsky, D., Burrows, A. \& Pinto, P. 2000, ApJ, 538, 885

Sun, Z.-P., Schubert, G. \& Glatzmeier, G.A. 1993, Science, 260, 661

Williams, P. K. G., Charbonneau, D., Cooper, C. S., Showman, A. P. \& Fortney, J. J. 2006, ApJ in press (astro-ph/0601092)

Winn, J. \& Holman, M. 2005, ApJ, 628, L159 
Table 1: Global Planetary Parameters

\begin{tabular}{|c|c|c|c|c|c|c|c|c|c|c|}
\hline Planet $^{(1)}$ & $\begin{array}{l}M_{\star}^{(2)} \\
\left(M_{\odot}\right)\end{array}$ & $\begin{array}{c}P_{\text {orb }}^{(3)} \\
\text { (days) }\end{array}$ & $\begin{array}{l}a^{(4)} \\
(\mathrm{AU})\end{array}$ & $e^{(5)}$ & $\begin{array}{l}M_{p}^{(6)} \\
\left(M_{\mathrm{J}}\right)\end{array}$ & $\begin{array}{c}R_{p}^{(7)} \\
(\mathrm{m})\end{array}$ & $\begin{array}{c}g^{(8)} \\
\left(\mathrm{m} \mathrm{s}^{-2}\right)\end{array}$ & $\begin{array}{c}\Omega^{(9)} \\
\left(\operatorname{rad~s}^{-1}\right)\end{array}$ & $\begin{array}{c}\bar{H}_{p}^{(10)} \\
(\mathrm{m})\end{array}$ & $\begin{array}{c}\bar{U}^{(11)} \\
\left(\mathrm{m} \mathrm{s}^{-1}\right)\end{array}$ \\
\hline Jupiter & 1.0 & $4,332.6$ & 5.2 & 0.0489 & 1.0 & $7.1 \times 10^{7}$ & 23 & $1.8 \times 10^{-4}$ & $2 \times 10^{4}$ & 70 \\
\hline Saturn & 1.0 & $10,759.2$ & 9.58 & 0.0565 & 0.3 & $6 \times 10^{7}$ & 9 & $1.6 \times 10^{-4}$ & $4 \times 10^{4}$ & 400 \\
\hline Uranus & 1.0 & $30,685.4$ & 19.2 & 0.0457 & 0.046 & $2.6 \times 10^{7}$ & 9 & $(-) 1 \times 10^{-4}$ & $3.5 \times 10^{4}$ & 300 \\
\hline Neptune & 1.0 & $60,189.0$ & 30.05 & 0.0113 & 0.054 & $2.5 \times 10^{7}$ & 11 & $9.75 \times 10^{-5}$ & $3 \times 10^{4}$ & 300 \\
\hline HD 209458 b & 1.05 & 3.5247 & 0.045 & 0.0 & 0.69 & $10^{8}$ & 8 & $2.1 \times 10^{-5(a)}$ & $7 \times 10^{5(b)}$ & - \\
\hline
\end{tabular}

NOTES: (1) Giant planets (2) Parent star mass (3) Orbital period (4) Semi-major axis (5) Eccentricity (6) Planet mass (7) Planet radius (8) Surface gravity (9) Rotation rate (10) Global pressure scale height (11) Global root mean square velocity scale (a) Assuming spin-orbit synchronization (b) From global radiative equilibrium. 
Table 2: Summary of Simulations Discussed

\begin{tabular}{|c|c|c|c|c|c|c|c|}
\hline Model & $\begin{array}{c}\bar{U}^{(1)} \\
\left(\mathrm{m} \mathrm{s}^{-1}\right)\end{array}$ & $\begin{array}{l}\bar{T}_{0}^{(2)} \\
(\mathrm{K})\end{array}$ & $\eta^{(3)}$ & $\begin{array}{l}L_{\mathcal{R}}{ }^{(4)} \\
\left(R_{p}\right)\end{array}$ & $\begin{array}{c}L_{\beta}{ }^{(5)} \\
\left(R_{p}\right)\end{array}$ & $\mathrm{N}_{\text {bands }}^{(6, \dagger)}$ & Remarks \\
\hline $\mathrm{S} 1$ & 70 & 130 & 0 & .03 & .23 & 14 & Jupiter \\
\hline $\mathrm{S} 2$ & 400 & 95 & 0 & .03 & .64 & 5 & Saturn ( $q$-map not shown) \\
\hline $\mathrm{S} 3$ & 300 & 60 & 0 & .11 & 1.1 & 3 & Uranus ( $q$-map not shown) \\
\hline $\mathrm{S} 4$ & 300 & 60 & 0 & .11 & 1.1 & 3 & Neptune \\
\hline $\mathrm{J} 1$ & 70 & 130 & 0 & .02 & .23 & 14 & S1 w/ different size initial stirring \\
\hline $\mathrm{J} 2$ & 70 & $130^{*}$ & 0 & .02 & .23 & 14 & $\mathrm{~J} 1 \mathrm{w} /$ shallow-water model \\
\hline U1 & 70 & 260 & 0 & .05 & .23 & 14 & "Warm" Jupiter \\
\hline $\mathrm{U} 2$ & 400 & 1450 & 0 & 1.2 & 1.2 & 3 & "Unsynchronized" HD 209458 b \\
\hline U3 & 400 & 1800 & 0 & 1.3 & 1.2 & 3 & "Warm" U2 \\
\hline $\mathrm{U} 4$ & 400 & 800 & 0 & 0.9 & 1.2 & 3 & "Cool" U2 \\
\hline $\mathrm{H} 1$ & 100 & 1450 & .0 & 1.2 & 0.6 & 5 & HD 209458 b, no thermal contrast \\
\hline $\mathrm{H} 2$ & 100 & 1450 & .02 & 1.2 & 0.6 & 5 & HD 209458 b, low thermal contrast \\
\hline H3 & 100 & 1450 & .04 & 1.2 & 0.6 & 5 & HD 209458 b, medium contrast \\
\hline $\mathrm{H} 4$ & 100 & 1450 & .20 & 1.2 & 0.6 & 5 & HD 209458 b, high thermal contrast \\
\hline $\mathrm{H} 5$ & 200 & 1450 & .04 & 1.2 & 0.8 & 4 & HD 209458 b, low speed jets \\
\hline $\mathrm{H} 6$ & 400 & 1450 & .04 & 1.2 & 1.2 & 3 & HD 209458 b, medium speed jets \\
\hline $\mathrm{H} 7$ & 1000 & 1450 & .04 & 1.2 & 1.8 & 2 & HD 209458 b, high speed jets \\
\hline $\mathrm{Z1}$ & 0 & 1450 & .04 & 1.2 & $1.8^{\ddagger}$ & 2 & H6 w/ zero initial vel. \& superrot. \\
\hline $\mathrm{Z} 2$ & 0 & 1450 & .04 & 1.2 & $1.8^{\ddagger}$ & 2 & Z2 w/o superrot. \\
\hline $\mathrm{Z3}$ & 0 & 1450 & .04 & 1.2 & $1.8^{\ddagger}$ & 2 & $\mathrm{Z} 1 \mathrm{w} /$ eddies \\
\hline
\end{tabular}

NOTES: (1) Initial global root mean square velocity (2) Global mean temperature at cloudtop for Solar System giant planets and fiducial cloudtop for extrasolar giant planets (3) Forcing amplitude (4) Nondimensional Rossby deformation radius at the pole, based on $\bar{H}_{p}(5)$ Nondimensional Rhines length at the equator, based on $\bar{U}(6)$ Number of zonal jets estimated, based on $L_{\beta}(\dagger)$ May eventually erode to 2 , if $L_{\mathcal{R}} \gtrsim 0.3(*)$ Based on layer thickness $(\ddagger)$ Based on $\bar{U}$ at quasi-equilibrium. 
Fig. 1. - Equivalent-barotropic turbulence simulation of Jupiter. Contour maps of the flow tracer, potential vorticity $q$, from six time frames are shown in orthographic view centered at the equator. Positive (negative) values are in full (dashed) contours; 40 contour levels are shown. Time, in unit of planetary rotation periods $(\tau=2 \pi / \Omega)$, is indicated in the upper left corner of each frame. Using only five physical parameters, which are known from observations, the initially random turbulent flow self-organizes into one dominated by zonal (east-west) bands - alternating, high/low $q$ gradients in the meridional (north-south) direction - similar to the actual Jupiter.

Fig. 2.- Simulation of Figure 1 in stereographic view. The boundary of the disk is the equator, and the center of the disk is the north pole. In this projection, the polar region occupies a smaller area of the disk compared with the equatorial region, as in Figure 1. A quasi-steady banded state is reached and is robust. The general flow picture does not change even at time, $\tau=2000$, the duration of this run. The interaction of the flow structures (vortices and jets) is weak, due to the extremely small Rossby deformation radius, $L_{\mathcal{R}}$ (see text for definition). Jets and vortices do not coalesce.

Fig. 3.- Quasi-steady zonal jet (positive eastward) profile from the simulation of Figures 1 and 2 at $\tau=300$. The jets are associated with the bands in Figures 1 and 2. Many qualitative features (number, width, and strengths of jets) of the observed profile on Jupiter are reproduced, giving confidence in the model approach. As in the shallow-water model calculations of Cho and Polvani (1996b), however, the sign of the equatorial jet is opposite to that observed. This is a common feature of one-layer turbulent models of Solar System giant planets without forcing. The observed prograde (eastward) equatorial jet is due to mechanism(s) not included in these unforced models.

Fig. 4.- Detailed comparison with the shallow-water model. Shallow-water simulation (a) and corresponding equivalent-barotropic simulation (b) are essentially identical. However, the equivalentbarotropic model is superior to the shallow-water model, since crucial parameters (e.g., the equivalent depth $H_{e}$ ) take on a more physical meaning. Note the presence of a large, stable anticyclone (a vortex, which is counter-rotating with respect to $\Omega$, which is anti-clockwise in the figure) at mid latitude [at $\sim 315^{\circ}$ in (a) and at $\sim 230^{\circ}$ longitude in (b) at $\sim 40^{\circ}$ latitude in both]. This structure is similar to Jupiter's Great Red Spot and is of approximately the same size and shape. The structure, along with the bands and jets, also appears in Figure 2, which has a different initial condition. Hence, these are robust features in our simulations.

Fig. 5.- Simulation of Neptune. In contrast to Jupiter (e.g., Figures 1 and 2), there is a large band of homogenized potential vorticity around the equator and a strong circumpolar vortex centered at each pole ( $\tau=146$ frame). A very small number of contours at low latitudes is present, compared with the high latitudes. The overall flow pattern is a direct consequence of the larger $\bar{U}$ and smaller $\Omega$ values (i.e., larger $L_{\mathcal{R}} / R_{p}$ and $L_{\beta} / R_{p}$, respectively), compared to those of Jupiter. 
Fig. 6. - Zonal jet profiles of the Solar System giant planets: (a) Jupiter, (b) Saturn, (c) Uranus, and (d) Neptune. The qualitative features of the jets, which are the principle structures of the large-scale circulation on the Solar System giant planets, are captured for all four planets. Only the observed parameters of Table 1 are used, and no explicit thermal forcing is imposed. The width of the jets roughly correspond to $L_{\beta}$. On all four planets, $L_{\mathcal{R}} / R_{p} \lesssim 1 / 3$, which allows the formed jets to be stable over long time. The sign of the equatorial jets on Jupiter and Saturn are reversed, compared with observations; but, the sign for Uranus and Neptune is as observed. The scale for Jupiter is different from that for the others.

Fig. 7.- A "warm" Jupiter, with an equilibrium temperature, $T_{e}=300 \mathrm{~K}$. Contour maps of the potential vorticity $(q)$ field at $\tau=199$ are shown in equatorial orthographic (a) and polar stereographic (b) views. The physical parameters used are identical to the simulation in Figures 1 and 2, except that $\overline{\mathcal{H}}$ is 2.3 times larger (see text for explanation). This case can serve as an example of a "Jupiter" which has migrated in to $\sim 1$ AU from the central star. Additionally, it could apply to a "cool", unsynchronized extrasolar giant planet with Jupiter $\Omega$ or to a level 2.3 pressure scale heights deeper than in previous models (compare with Figures 1 and 2). Due to the increased $L_{\mathcal{R}}$ value, the bands and jets are less pronounced, and the equatorial jet amplitude is reduced. There are also fewer vortices in the polar region.

Fig. 8. - Simulation of a HD 209458 b-like, unsynchronized close-in extrasolar giant planet in polar stereographic view: early evolution. The rotation rate for this planet is that of HD $209458 \mathrm{~b}$ (i.e., 8.4 times slower than that of Jupiter). The global average root mean square velocity $\bar{U}$ for HD $209458 \mathrm{~b}$ is not known and can range plausibly between $\sim 10^{2} \mathrm{~m} \mathrm{~s}^{-1}$ to $\sim 10^{3} \mathrm{~m} \mathrm{~s}^{-1}$. In this simulation, $\bar{U}=400 \mathrm{~m} \mathrm{~s}^{-1}$, roughly the maximum value of all the planets in the Solar System. We have varied this parameter in our study and summarize the findings in $\S 5$. The salient feature here is that the vortices and jets in the flow are much more dynamic compared with those in Figure 2, and even with those in Figure 5. This is due to the larger $L_{\mathcal{R}}$ value, which is $\sim R_{p}$ in this case.

Fig. 9.- Same simulation and view of Figure 8: evolution post equilibrium. By $\tau=70$ a broad band of smoothly varying $q$ forms outside a robust polar vortex, which has formed through continuous mergers. The flow is more similar to that on Neptune than on Jupiter (compare with Figures 2 and 5). As in Neptune, a polar vortex forms in each hemisphere, but the vortex is not centered on the pole. Robust, off-pole vortices are general features in simulations of the circulation of HD 209458 b. Another difference, consistent with less homogenized equatorial band, is the planetary (Rossby) wave breaking that appears more pronounced at mid-latitudes. 
Fig. 10.- Quasi-steady jet profiles corresponding to the simulation of Figures 8 and 9 (a) and the same simulation with a different $\bar{U}$, which is $100 \mathrm{~m} \mathrm{~s}^{-1}$ (b). The low number of jets is also a robust feature of our synchronized HD $209458 \mathrm{~b}$ simulations. The precise profile of the jets depends on the value of $\bar{U}$, and the profile is slightly time-varying in a given run. With a value of $\bar{U}$ characteristic of most Solar System giant planets, three or four broad jets form. When $\bar{U} \lesssim 100 \mathrm{~m} \mathrm{~s}^{-1}$ more jets may form. Occasionally a two-jet profile forms for $\bar{U} \gtrsim 400 \mathrm{~m} \mathrm{~s}^{-1}$ and also for $\bar{U}=0$ (see Figure 17). Note that in (b) the equatorial jet is prograde, demonstrating that a retrograde equatorial jet is not a necessary outcome of single-layer simulations. That outcome requires a small $L_{\mathcal{R}}$ value.

Fig. 11. - Unsynchronized HD 209458 b simulations with different equilibrium temperatures, $\bar{T}_{0}$ : (a) $1800 \mathrm{~K}$ and (b) $800 \mathrm{~K}$. The two temperatures are chosen to roughly bracket a variety of physical situations (e.g., distance, stellar flux, and albedo). For fixed flux and albedo, the higher $\bar{T}_{0}$ value can also represent an HD 209458 b-like planet which is $~ .005$ AU closer to the host star than HD 209458 b is to its star. Similarly, the lower $\bar{T}_{0}$ value can represent an HD 209458 b-like planet that is $\sim 0.15 \mathrm{AU}$ further away. The higher $\bar{T}_{0}$ value leads to a more smoothly varying low- to mid-latitude potential vorticity distribution, while the lower $\bar{T}_{0}$ value leads to much more planetary (Rossby) wave activity, along with a more centered polar vortex. The basic three-jet profile is not changed in either case.

Fig. 12. - HD $209458 \mathrm{~b}$ atmospheric flow for different forcing amplitudes, $\eta$ : (a) 0., (b) .02, (c) .04, and (d) .20. All other parameters in (a)-(d) are identical; in particular, $\bar{U}=100 \mathrm{~m} \mathrm{~s}^{-1}$. Formally, $\eta$ can range from 0 to $1-$ i.e., from no asymmetric forcing to full forcing, respectively. The latter corresponds to a temperature deviation, $\delta T=\mathcal{O}\left(\bar{T}_{0}\right)$, which is not physically realistic on large scales. The primary effect of increasing the forcing asymmetry is to sharpen the midlatitude jet $(\mathrm{a}-\mathrm{c})$. When the forcing is large, it induces large zonal asymmetry in the jet, destabilizing it and causing waves to break at high latitudes (d). A simulation with $\eta=0.4$ shows a flow that is basically a more violent version of $(d)$.

Fig. 13. - Temperature distribution, $T_{0}=T_{0}(\lambda, \phi)$, of the runs in Figure 12 with $\eta$ : (a) 0., (b) .02, (c) .04, and (d) .20. For a fixed $\bar{U}, T_{0}$ is strongly dependent on $\eta$. With no or low-amplitude forcing, $T_{0}$ essentially tracks the flow structure (a). As the forcing amplitude increases, however, there is a competition between the flow-induced and the forcing-induced temperature patterns: temperature anomalies either rotate quasi-steadily about the pole (b) or oscillate aperiodically about a point near the substellar point (c). For high $\eta$ values, the forcing dominates, and $T_{0}$ is essentially the day-night distribution centered on the substellar point (d). In each case, a cyclone/anticyclone pair at high latitudes is present (i.e., independent of the amplitude) and is a robust feature of our simulations (see Figures 12 and 15). 
Fig. 14. - HD 209458b circulation for different global root mean square velocities, $\bar{U}$ : (a) $100 \mathrm{~m} \mathrm{~s}^{-1}$, (b) $200 \mathrm{~m} \mathrm{~s}^{-1}$, (c) $400 \mathrm{~m} \mathrm{~s}^{-1}$, and (d) $1000 \mathrm{~m} \mathrm{~s}^{-1}$. The primary effect of larger $\bar{U}$ values is to strengthen and enlarge the polar vortex at high latitudes. At low to mid latitudes, breaking of planetary (Rossby) waves appear to be reduced when $\bar{U}$ is larger. However, in reality, the breaking also increases, as shown in Figure 15. The increase in vortex size is due to the initially more energetic vortices, which coalesce to form the final vortex. The vortex in (d) is large enough to nearly cap the planet, poleward of 60 degrees latitude, if it were centered at the pole.

Fig. 15. - Flow outside the polar vortex and global jet profile (for two different $\bar{U}$ values). Three same time frames of the simulation in Figure 14d are shown in $(\mathrm{a}-\mathrm{c})$. There are 50 contours in each map. Note the dominance of the cyclonic polar vortex (a) and the presence of a weaker, anticyclonic vortex, which is revealed when a smaller contour range is used (b). The anticyclone forms from high-amplitude breaking flow outside the cyclonic vortex (c). The cyclone is associated with a coherent cold region, and the anticyclone is associated with a coherent warm region-as shown in Figure 16. Zonal jet profiles in two runs with different $\bar{U}$ values: (d) $100 \mathrm{~m} \mathrm{~s}^{-1}$ and (e) $1000 \mathrm{~m} \mathrm{~s}^{-1}$. The number and width of the jets is qualitatively consistent with the Rhines scale, $L_{\beta}$.

Fig. 16. - HD $209458 \mathrm{~b}$ atmospheric temperature distribution $T_{0}$ for different global root mean square velocity $\bar{U}$ : (a) $100 \mathrm{~m} \mathrm{~s}^{-1}$, (b) $200 \mathrm{~m} \mathrm{~s}^{-1}$, (c) $400 \mathrm{~m} \mathrm{~s}^{-1}$, and (d) $1000 \mathrm{~m} \mathrm{~s}^{-1}$. The same simulations as in Figure 14 are shown. With forcing amplitude $\eta$ fixed, $T_{0}$ is strongly dependent on $\bar{U}$. At low $\bar{U}$ values, the forcing dominates and $T_{0}$ is essentially the day-night distribution centered on the substellar point (a). As $\bar{U}$ increases, competition between the forcing-induced and the flow-induced temperature patterns develops: temperature anomalies oscillate aperiodically about a point near the substellar point (b) or rotate quasi-steadily about the pole (c). At large $\bar{U}$ values, $T_{0}$ essentially tracks the flow structure on a several-days timescale (d).

Fig. 17.- Simulation starting at rest. The model is augmented to include the baroclinic effect of large-amplitude, zonally-asymmetric, equatorial forcing. Potential vorticity $(q)$ contours in cylindrical projection, centered at the substellar point, are shown for three time frames $(\mathrm{a}-\mathrm{c})$. Corresponding zonal jet profiles are shown to the right of each $q$-map. The forcing excites planetary (Rossby) waves (a). Angular momentum is transported as propagating planetary waves interact with the background flow (b). Eventually, the flow becomes very complex, at the high resolution of this simulation (c). However, the north-south symmetry is maintained throughout, indicating the dominance of the forcing. 
Fig. 18. - Summary of results with quiescent initial condition. The plotting is identical to Figure 17. Late-time results in three simulations with different forcing conditions are shown: (a) day-night thermal forcing only, (b) with angular momentum forcing added, and (c) with initial random stirring added (i.e. atmosphere no longer at rest initially). With only day-night forcing applied, the flow is laminar and consistent with $q$ lines distorted by the thermal forcing. Finally, with angular momentum forcing also added, the flow becomes more complex and the atmosphere superrotates. With the addition of initial random stirring, the flow becomes "eddy driven", if the stirring is energetic. The equatorial jet is weakly retrograde in this case. 
This figure "fig1.gif" is available in "gif" format from: http://arxiv.org/ps/astro-ph/0607338v1 
This figure "fig2.gif" is available in "gif" format from: http://arxiv.org/ps/astro-ph/0607338v1 
This figure "fig3.gif" is available in "gif" format from: http://arxiv.org/ps/astro-ph/0607338v1 
This figure "fig4.gif" is available in "gif" format from: http://arxiv.org/ps/astro-ph/0607338v1 
This figure "fig5.gif" is available in "gif" format from: http://arxiv.org/ps/astro-ph/0607338v1 
This figure "fig6.gif" is available in "gif" format from: http://arxiv.org/ps/astro-ph/0607338v1 
This figure "fig7.gif" is available in "gif" format from: http://arxiv.org/ps/astro-ph/0607338v1 
This figure "fig8.gif" is available in "gif" format from: http://arxiv.org/ps/astro-ph/0607338v1 
This figure "fig9.gif" is available in "gif" format from: http://arxiv.org/ps/astro-ph/0607338v1 
This figure "fig10.gif" is available in "gif" format from: http://arxiv.org/ps/astro-ph/0607338v1 
This figure "fig11.gif" is available in "gif" format from: http://arxiv.org/ps/astro-ph/0607338v1 
This figure "fig12.gif" is available in "gif" format from: http://arxiv.org/ps/astro-ph/0607338v1 
This figure "fig13.gif" is available in "gif" format from: http://arxiv.org/ps/astro-ph/0607338v1 
This figure "fig14.gif" is available in "gif" format from: http://arxiv.org/ps/astro-ph/0607338v1 
This figure "fig15.gif" is available in "gif" format from: http://arxiv.org/ps/astro-ph/0607338v1 
This figure "fig16.gif" is available in "gif" format from: http://arxiv.org/ps/astro-ph/0607338v1 
This figure "fig17.gif" is available in "gif" format from: http://arxiv.org/ps/astro-ph/0607338v1 
This figure "fig18.gif" is available in "gif" format from: http://arxiv.org/ps/astro-ph/0607338v1 